\title{
Carbenoid Chain Reactions: Substitutions by Organolithium Compounds at Unactivated 1-Chloro- 1-alkenes
}

Rudolf Knorr, ${ }^{*, \neq}$ Claudio Pires, Claudia Behringer, Thomas Menke, Johannes Freudenreich, Eva C. Rossmann, and Petra Böhrer

Department of Chemistry and Biochemistry, Ludwig-Maximilians-Universität, Butenandtstr. 5-13, 81377 München, Germany

E-mail: rhk@cup.uni-muenchen.de

\section{Contents:}

1. General Experimental Procedures.

2. Preparatory Synthetic Studies: 12a, 12c, 15, 4- $\left(\mathrm{Me}_{3} \mathrm{Si}\right) \mathrm{C}_{6} \mathrm{H}_{4} \mathrm{Br}$, and $2,4,6-(t-\mathrm{Bu})_{3} \mathrm{C}_{6} \mathrm{H}_{2} \mathrm{Cl}$ (ClMes*).

3. Substitution Products (4, 10i, 16, and 20), ${ }^{13} \mathrm{C}$ NMR spectra, and NMR Assignments.

4. Side-products $\mathbf{S 7}$ - S10 Formed from 2-(1-Chloroethylidene)-1,1,3,3-tetramethylindan (4f).

5. Substitutions with $t$-BuC $\equiv C L i$ : $\mathbf{S 1 1}-\mathbf{S 1 5}$.

6. Kinetic H/D Isotope Effect in the Competition of Reagents $\mathbf{2 a}$ and $\mathbf{2 b}$ for MeLi.

7. References

Note: Formulae, Schemes, and literature references taken over from the Main Text are utilized with their unchanged numbers, whereas those cited here for the first time are denominated beginning with S. 
1. General Experimental Procedures. Organolithium compounds were handled under a stream of dry argon cover gas. Experiments in NMR tubes $(5 \mathrm{~mm})$ were performed with nondeuterated solvents $\left(\approx 0.7 \mathrm{~mL}\right.$, containing $\approx 0.035 \mathrm{~mL}$ of $\mathrm{C}_{6} \mathrm{D}_{6}$ or $\mathrm{C}_{6} \mathrm{D}_{12}$ if required as "lock substances"); this allowed product analyses to be carried out in situ before workup. Concentrations were estimated by comparison with the ${ }^{1} \mathrm{H}$ NMR integral of a sealed capillary filled with pure $\mathrm{ClCH}_{2} \mathrm{C} \equiv \mathrm{N}\left(\delta_{\mathrm{H}} \approx 3.9\right)$ or of the low-field ${ }^{13} \mathrm{C}$ satellites of the solvents. Hydrogen versus deuterium distributions were determined by pairwise integrations of the baseline separated NMR absorptions having sufficiently large isotope-induced shift differences, with the machine parameters set for maximum resolution [for example, number of points $n p=160000\left({ }^{1} \mathrm{H}\right.$ at $\left.400 \mathrm{MHz}\right)$ or $524288\left({ }^{13} \mathrm{C}\right)$, and acquisition times at $\left.\approx 13 \mathrm{sec}\right]$. Commercially available solutions of methyllithium $\left(\delta_{\mathrm{H}} \approx-2\right)$ in $\mathrm{Et}_{2} \mathrm{O}$ containing $\mathrm{LiBr}$, of $n$-butyllithium in hexanes $\left(\delta_{\mathrm{H}} \approx-0.75\right.$ in benzene), and of tert-butyllithium $(t-\mathrm{BuLi})$ in pentane were used. The $\mathrm{Br} / \mathrm{Li}$ interchange reaction with ensuing $\beta$-elimination of $\mathrm{HBr}(t-\mathrm{BuLi}+\mathrm{ArBr} \rightarrow t-\mathrm{BuBr}+\mathrm{ArLi}, t-\mathrm{BuLi}+t-\mathrm{BuBr} \rightarrow t$ $\mathrm{BuH}+\mathrm{LiBr}+\mathrm{Me}_{2} \mathrm{C}=\mathrm{CH}_{2}$ ) was employed in ethereal solvents to prepare solutions of phenyllithium $\left(\delta_{\mathrm{H}} \approx 8.0\right.$ for two $o-\mathrm{H}$ ), $21 \mathrm{~m}$, and $\mathbf{2 1 \mathbf { n }}$. However, $21 \mathbf{p}$ could be prepared best with $n$-BuLi in place of $t$ BuLi because it precipitated from its solution in THF/hexanes $(1: 4)^{\mathrm{S1}}$ immediately.

2. Preparatory Synthetic Studies: 12a, 12c, 15, 4- $\left(\mathrm{Me}_{3} \mathrm{Si}\right) \mathrm{C}_{6} \mathrm{H}_{4}, \mathrm{Br}$, and $2,4,6-(t-\mathrm{Bu})_{3} \mathrm{C}_{6} \mathrm{H}_{2} \mathrm{Cl}$ (ClMes*).

\section{Scheme 2}

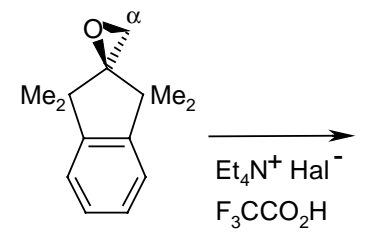

11

$\uparrow$

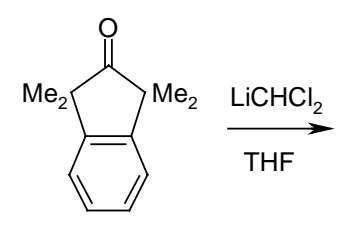

14

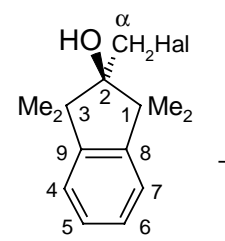

12a: $\mathrm{Hal}=\mathrm{Cl}$

b: $\mathrm{Hal}=\mathrm{Br}$

c: $\mathrm{Hal}=\mathrm{I}$

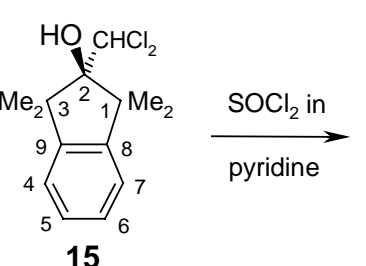

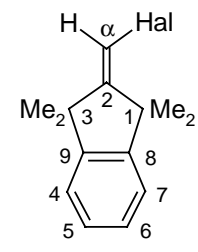

13: $\mathrm{Hal}=\mathrm{Br}$

2a: $\mathrm{Hal}=\mathrm{Cl}$

t-BuMgCl

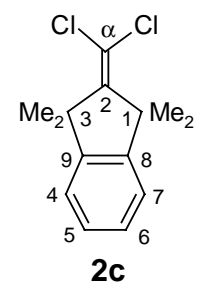

Nucleophilic iodination of the oxirane $\mathbf{1 1}$ to afford 12c (Scheme 2) was easily achieved, but all attempts to eliminate water from $\mathbf{1 2 c}$ were unsuccessful. 
2-Chloromethyl-1,1,3,3-tetramethyl-2-indanol (12a). Tetraethylammonium chloride (1.15 g, 6.92 mmol) in chloroform $(5 \mathrm{~mL})$ was dried overnight with a few small pieces of $\mathrm{CaCl}_{2}$. The dry solution was quickly decanted under dry argon cover gas into a dry Schlenk flask $(25 \mathrm{~mL})$ equipped with a reflux condenser and a drying tube. After addition of the oxirane ${ }^{9} \mathbf{1 1}(300 \mathrm{mg}, 1.48 \mathrm{mmol})$ and trifluoroacetic acid $(0.21 \mathrm{~mL}, 2.74 \mathrm{mmol})$, the mixture was heated to reflux for four hours and then cooled, washed with water (no $\mathrm{NaOH}$ !) until neutral, and dried over $\mathrm{Na}_{2} \mathrm{SO}_{4}$. Removal of the solvent furnished $230 \mathrm{mg}(65 \%)$ of the almost uncontaminated, solid alcohol 12a. After two recrystallizations from pentane, the mp was $87-88{ }^{\circ} \mathrm{C}$. It is important to avoid the contact of 12a with strong alkali because this would cause recyclization to the oxirane 11. - ${ }^{1} \mathrm{H} \mathrm{NMR}\left(400 \mathrm{MHz}, \mathrm{CDCl}_{3}\right) \delta 1.32$ (s, 1 /3- $\mathrm{CH}_{3}$ cis to $\mathrm{CH}_{2} \mathrm{Cl}$ ), 1.45 (s, 1-/3- $\mathrm{CH}_{3}$ trans to $\left.\mathrm{CH}_{2} \mathrm{Cl}\right), 2.43$ (s, OH), $3.95\left(\mathrm{~s}, \mathrm{CH}_{2} \mathrm{Cl}\right), 7.14$ (m, 4-,7$\mathrm{H}), 7.23(\mathrm{~m}, 5-/ 6-\mathrm{H}) ;{ }^{13} \mathrm{C} \mathrm{NMR}\left(100.6 \mathrm{MHz}, \mathrm{CDCl}_{3}\right) \delta 23.73\left(\mathrm{qq},{ }^{1} J=126.9 \mathrm{~Hz},{ }^{3} J=4.9 \mathrm{~Hz}, 1-/ 3-\right.$ $\mathrm{CH}_{3}$ trans to $\left.\mathrm{CH}_{2} \mathrm{Cl}\right), 28.84\left(\mathrm{qq},{ }^{1} \mathrm{~J}=126.9 \mathrm{~Hz},{ }^{3} \mathrm{~J}=4.9 \mathrm{~Hz}, 1-/ 3-\mathrm{CH}_{3}\right.$ cis to $\left.\mathrm{CH}_{2} \mathrm{Cl}\right), 49.82\left(\mathrm{t},{ }^{1} \mathrm{~J}=\right.$ $\left.150.0 \mathrm{~Hz}, \mathrm{CH}_{2} \mathrm{Cl}\right), 50.53\left(\mathrm{~m}, \mathrm{C}^{1,3}\right), 84.21\left(\mathrm{~m}, \mathrm{C}^{2}\right), 122.41\left(\mathrm{dm},{ }^{1} J=157.1 \mathrm{~Hz}, \mathrm{C}^{4,7}\right), 127.46\left(\mathrm{ddd},{ }^{1} J=\right.$ $\left.159.1 \mathrm{~Hz},{ }^{3} J=7.4 \mathrm{~Hz}, \mathrm{C}^{5,6}\right), 148.52\left(\mathrm{~m}, \mathrm{C}^{8,9}\right)$; IR (KBr) 3581 and 3567 (2 sharp O-H), 2962, 2879, 1482, 1450, 1366, 1074, and $760 \mathrm{~cm}^{-1}$. Anal. Calcd for $\mathrm{C}_{14} \mathrm{H}_{19} \mathrm{ClO}$ (238.8): C, 70.43; H, 8.02; $\mathrm{Cl}$, 14.85. Found: C, 70.67; H, 7.76; Cl, 14.27.

NMR Assignments: Molecular mechanics calculations (PC-Model) indicated that the $\mathrm{CH}_{2} \mathrm{Cl}$ group prefers a quasi-equatorial position at the five-membered ring, with dihedral angles $\mathrm{Cl}-\mathrm{CH}_{2}-\mathrm{C}^{2}-\mathrm{O}=$ $53^{\circ}$ and $\mathrm{H}-\mathrm{O}-\mathrm{C}^{2}-\mathrm{CH}_{2}=51^{\circ}$. Consequently, $\mathrm{CH}_{2} \mathrm{Cl}$ showed crosspeaks in the NOESY $\left({ }^{1} \mathrm{H},{ }^{1} \mathrm{H}\right)$ spectrum with both the cis-1-/3- $\mathrm{CH}_{3}$ (stronger, quasi-axial, upfield) and the trans-1-/3- $\mathrm{CH}_{3}$ protons (weaker, quasi-equatorial, downfield). This geometry was also in accord with the NOESY correlations $\mathrm{OH} \leftrightarrow$ trans-1-/3- $\mathrm{CH}_{3} \leftrightarrow 4-/ 7-\mathrm{H}$. On this basis, the signals of the cis (downfield) and trans (upfield) methyl carbon nuclei were assigned by selective decoupling of the methyl protons. The resonances of $\mathrm{C}^{4,7}$ are known ${ }^{58}$ to be always upfield from those of $\mathrm{C}^{5,6}$.

2-Iodomethyl-1,1,3,3-tetramethyl-2-indanol (12c). A chloroform solution (7.20 $\mathrm{mL})$ of the oxirane $^{9} \mathbf{1 1}$ (400 mg, $\left.1.98 \mathrm{mmol}\right)$ and well-dried tetraethylammonium iodide (2.08 g, $\left.8.10 \mathrm{mmol}\right)$ was heated to reflux for $3.5 \mathrm{~h}$ with trifluoroacetic acid $(0.30 \mathrm{~mL}, 3.92 \mathrm{mmol})$ under argon. The mixture was washed with distd. water until neutral, dried over $\mathrm{Na}_{2} \mathrm{SO}_{4}$, and concentrated to give $590 \mathrm{mg}$ of crude 12c, contaminated with olefin $\mathbf{4 a}(7 \%)$ and, as usual, ${ }^{9}$ with 1,2,3,3-tetramethylindene (19\%). These side-products were removed by dissolving the mixture in pentane, transfering the soluble portion to a silica column, elution with petroleum ether, and distillation at $100-115^{\circ} \mathrm{C}$ (bath temp.)/0.2 mbar to furnish $153 \mathrm{mg}(23 \%)$ of pure 12c. Colorless plates, mp $66-67{ }^{\circ} \mathrm{C}$ (cooled pentane); ${ }^{1} \mathrm{H}$ NMR (400 $\left.\mathrm{MHz}, \mathrm{CDCl}_{3}\right) \delta 1.33\left(\mathrm{~s}, 1-/ 3-\mathrm{CH}_{3}\right.$ cis to $\left.\mathrm{CH}_{2} \mathrm{I}\right), 1.47$ (s, 1-/3- $\mathrm{CH}_{3}$ trans to $\mathrm{CH}_{2} \mathrm{I}$ ), 2.03 (s, $\mathrm{OH}$ ), 3.65 (s, $\left.\mathrm{CH}_{2} \mathrm{I}\right), 7.14(\mathrm{~m}, 4-/ 7-\mathrm{H}), 7.23(\mathrm{~m}, 5-/ 6-\mathrm{H}) ;{ }^{13} \mathrm{C} \mathrm{NMR}\left(100.6 \mathrm{MHz}, \mathrm{CDCl}_{3}\right) \delta 14.37\left(\mathrm{CH}_{2} \mathrm{I}\right), 23.71(1-$ 
13- $\mathrm{CH}_{3}$ trans to $\left.\mathrm{CH}_{2} \mathrm{I}\right), 29.06\left(1-/ 3-\mathrm{CH}_{3}\right.$ cis to $\left.\mathrm{CH}_{2} \mathrm{I}\right), 50.92\left(\mathrm{~m}, \mathrm{C}^{1,3}\right), 82.67\left(\mathrm{C}^{2}\right), 122.41\left(\mathrm{C}^{4,7}\right), 127.49$ $\left(\mathrm{C}^{5,6}\right), 148.69\left(\mathrm{C}^{8,9}\right)$, assigned by comparison with the very similar spectra of chloride 12a; IR (KBr) 3571 (sharp O-H), 2984, 2960, 2870, 1482, 1450, and 754 (s) $\mathrm{cm}^{-1}$. Anal. Calcd for $\mathrm{C}_{14} \mathrm{H}_{19} \mathrm{IO}$ (330.2): C, 50.92; H, 5.80; I, 38.43. Found: C, 51.03; H, 5.58; I, 38.69.

Although 12c was stable in pyridine solution, it was reduced quickly to olefin $4 \mathbf{a}$ upon the addition of thionyl chloride (1.8 or 6 equiv) to this solution. The desired dehydration was also not achieved on treatment of $12 \mathrm{c}$ with oxalyl chloride in $\mathrm{CCl}_{4}$ with or without $\mathrm{DMF} / \mathrm{NEt}_{3}$ up to $+65{ }^{\circ} \mathrm{C}$, or with acetyl chloride in $\mathrm{CCl}_{4}$ up to $+80{ }^{\circ} \mathrm{C}, \mathbf{1 2 c}$ being reisolated in every case. Unidentified product mixtures resulted from the slow reaction of $\mathbf{1 2 c}$ with acetic anhydride at up to $+140{ }^{\circ} \mathrm{C}$, or with acetyl chloride and 4-dimethylaminopyridine in refluxing $\mathrm{CCl}_{4}$, or from treatment of the olefin $\mathbf{4 a}$ with elemental iodine.

\section{2-(Dichloromethyl)-1,1,3,3-tetramethyl-2-indanol (15). 1,1,3,3-Tetramethyl-2-indanone ${ }^{8}$}

$12.00 \mathrm{~g}, 63.74 \mathrm{mmol})$, anhydrous THF $(100 \mathrm{~mL})$, and anhydrous dichloromethane $(12.3 \mathrm{~mL}, 191.2$ mmol) were placed in a dry three-necked flask $(500 \mathrm{~mL})$ fitted with a thermometer, dropping funnel $(250 \mathrm{~mL})$, magnetic stirring bar, and an inlet connected to a slow stream of dry argon cover gas. The flask was cooled to $-50{ }^{\circ} \mathrm{C}$ and its contents stirred during the dropwise addition (45 min) of lithium diisopropylamide in THF solution, which had been prepared from diisopropylamine (29.6 mL, 210 mmol), anhydrous THF $(50 \mathrm{~mL})$, and $n$-butyllithium in hexane $(79.6 \mathrm{~mL}, 191 \mathrm{mmol})$ at below $+9^{\circ} \mathrm{C}$. Stirring was continued for $90 \mathrm{~min}$ during warm-up to an internal temperature of $-20{ }^{\circ} \mathrm{C}$ and a subsequent re-cooling to $-40{ }^{\circ} \mathrm{C}$. The temperature rose to $-20{ }^{\circ} \mathrm{C}$ during the dropwise addition of acetic acid ( $20 \mathrm{~mL}$ dissolved in $75 \mathrm{~mL}$ of THF), and there was the formation of a voluminous white precipitate. The flask was warmed up until its contents could be poured onto $2 \mathrm{M} \mathrm{HCl}(500 \mathrm{~mL}$, dissolving the precipitate), and the solution was then extracted with $\mathrm{Et}_{2} \mathrm{O}(400 \mathrm{~mL}$, then $2 \times 200 \mathrm{~mL})$. The combined ethereal extracts were washed with distd. water (no alkali!) until neutral, dried over $\mathrm{MgSO}_{4}$, and concentrated to give $15.67 \mathrm{~g}(90 \%)$ of the only marginally contaminated alcohol $\mathbf{1 5}$ as a brown powder. Recrystallization form low-boiling petroleum ether $(150 \mathrm{~mL})$ furnished $11.81 \mathrm{~g}(68 \%)$ of spectroscopically pure, brown crystals with $\mathrm{mp} 138-141^{\circ} \mathrm{C}$. The analytically pure, colorless crystals were obtained through repeated crystallization and had mp $146-147.5^{\circ} \mathrm{C} ;{ }^{1} \mathrm{H} \mathrm{NMR}\left(400 \mathrm{MHz}, \mathrm{CDCl}_{3}\right)$ $\delta 1.43$ (s, 1-/3- $\mathrm{CH}_{3}$ cis to $\mathrm{CHCl}_{2}$ ), 1.57 (s, 1-/3- $\mathrm{CH}_{3}$ trans to $\mathrm{CHCl}_{2}$ ), 2.46 (sharp s, exchangeable with $\left.\mathrm{D}_{2} \mathrm{O}, \mathrm{OH}\right), 6.26\left(\mathrm{~s}, \mathrm{CHCl}_{2}\right), 7.11(\mathrm{~m}, 4-/ 7-\mathrm{H}), 7.22(\mathrm{~m}, 5-/ 6-\mathrm{H}) ;{ }^{13} \mathrm{C} \mathrm{NMR}\left(100.6 \mathrm{MHz}, \mathrm{CDCl}_{3}\right) \delta 23.7$ (qq, ${ }^{1} J=127.0 \mathrm{~Hz},{ }^{3} J=4.7 \mathrm{~Hz}, 1-/ 3-\mathrm{CH}_{3}$ trans to $\mathrm{CHCl}_{2}$ ), 28.2 (qq, ${ }^{1} J=127.0 \mathrm{~Hz},{ }^{3} \mathrm{~J}=4.7 \mathrm{~Hz}, 1-/ 3-$ $\mathrm{CH}_{3}$ cis to $\mathrm{CHCl}_{2}$ ), 52.1 (unresolved $\mathrm{m}, \mathrm{C}^{1,3}$ ), 79.0 (sharp d, ${ }^{1} \mathrm{~J}=174.1 \mathrm{~Hz}, \mathrm{CHCl}_{2}$ ), 87.9 (unresolved $\left.\mathrm{m}, \mathrm{C}^{2}\right), 122.3\left(\mathrm{dm},{ }^{1} J=158 \mathrm{~Hz}, \mathrm{C}^{4,7}\right), 127.7\left(\mathrm{ddd},{ }^{1} J=160 \mathrm{~Hz},{ }^{3} J=7.3 \mathrm{~Hz}, \mathrm{C}^{5,6}\right), 148.0\left(\mathrm{~m}, \mathrm{C}^{8,9}\right)$, assigned by comparison with the monochloride 12a; IR (KBr) 3533 and 3487 ( 2 sharp $\mathrm{O}-\mathrm{H}), 3010$, 
2985, 2963, 2873, 1482, 1386, 1371, 1088, 1077, 784, 768, 760, 744, and $734 \mathrm{~cm}^{-1}$. Anal. Calcd for $\mathrm{C}_{14} \mathrm{H}_{18} \mathrm{Cl}_{2} \mathrm{O}$ (273.2): C, 61.55; H, 6.64; Cl, 25.95. Found: C, 61.59; H, 6.72; Cl, 26.63.

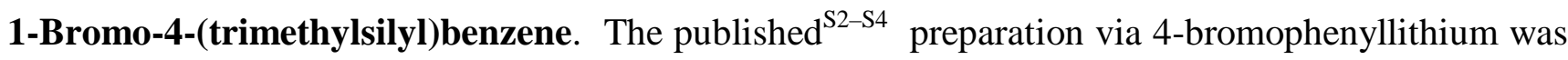
modified by the employment of $n$-BuLi ${ }^{\mathrm{S4}, \mathrm{S} 5}$ at $-30{ }^{\circ} \mathrm{C}$. A dried Schlenk flask $(250 \mathrm{~mL})$ was charged with 1,4-dibromobenzene $(10.62 \mathrm{~g}, 45.02 \mathrm{mmol})$ in anhydrous $\mathrm{Et}_{2} \mathrm{O}(80 \mathrm{~mL})$ and then cooled at $-30{ }^{\circ} \mathrm{C}$ with stirring under argon cover gas. After the dropwise addition (30 min) of $n$-BuLi (49.4 mmol) in hexane $(20.60 \mathrm{~mL})$, the mixture was cooled in an ice bath and treated with chlorotrimethylsilane $(6.25$ $\mathrm{mL}, 49.5 \mathrm{mmol})$. After further stirring for $30 \mathrm{~min}$, the contents were poured into distd. water (100 $\mathrm{mL})$. The separated aqueous layer was extracted with $\mathrm{Et}_{2} \mathrm{O}(3 \times 50 \mathrm{~mL})$, and the combined $\mathrm{Et}_{2} \mathrm{O}$ phases were washed until neutral, dried over $\mathrm{MgSO}_{4}$, and concentrated to furnish $9.918 \mathrm{~g}(96 \%)$ of the almost pure product $\left({ }^{1} \mathrm{H}\right.$ NMR as in ref S4). The alternative preparation via 4-bromophenylmagnesium bromide $^{\mathrm{S} 2, \mathrm{~S} 6, \mathrm{~S} 7}$ was reported $^{\mathrm{S} 2}$ to produce a significant admixture of 1,4-bis(trimethylsilyl)benzene.

Independent Preparation of Chloro-2,4,6-tri-tert-butylbenzene (CIMes*). An independently prepared sample of this byproduct of $\mathbf{4 p}$ was required for the identification of its NMR signals in the crude product mixtures. It was obtained by the addition of isopentyl nitrite to a mixture of 2,4,6-tritert-butylaniline $\left(\mathrm{H}_{2} \mathrm{NMes}^{*}\right)$ and $\mathrm{BrCCl}_{3}$ in 1,2-dimethoxyethane at $60-75{ }^{\circ} \mathrm{C}$. ${ }^{\mathrm{S} 8}$ The glistening platelets, recrystallized from ethanol in $14 \%$ yield, had mp $153-155{ }^{\circ} \mathrm{C}$ (ref S9: $156-158{ }^{\circ} \mathrm{C}$; $\operatorname{ref} \mathrm{S} 10$ : 156.5-160 ${ }^{\circ} \mathrm{C}$; $\left.\operatorname{ref} \mathrm{S} 11: 162{ }^{\circ} \mathrm{C}\right) .{ }^{1} \mathrm{H} \mathrm{NMR}\left(400 \mathrm{MHz}, \mathrm{CDCl}_{3}\right) \delta 1.31(\mathrm{~s}, 4-t-\mathrm{Bu}), 1.53(\mathrm{~s}, 2-/ 6-t-\mathrm{Bu})$, $7.38(\mathrm{~s}, 3-/ 5-\mathrm{H})$ as in ref S12 (compare ref S11); ${ }^{13} \mathrm{C} \mathrm{NMR}\left(100.6 \mathrm{MHz}, \mathrm{CDCl}_{3}\right) \delta 30.5\left(2-/ 6-\mathrm{CMe}_{3}\right)$, $31.4\left(4-\mathrm{CMe}_{3}\right), 34.9\left(4-\mathrm{CMe}_{3}\right), 37.2\left(2-/ 6-C \mathrm{Me}_{3}\right), 123.2\left(\mathrm{C}^{3,5}\right), 131.3\left(\mathrm{C}^{4}\right), 147.0\left(\mathrm{C}^{2,6}\right), 147.8\left(\mathrm{C}^{1}\right)$, compare ref S12; IR (KBr) 2963, 2872, 1594, 1476, 1411, 1365 (s), 1221, 1038, and $879 \mathrm{~cm}^{-1}$. Anal. Calcd for $\mathrm{C}_{18} \mathrm{H}_{29} \mathrm{Cl}$ (280.9): C, 76.97; H, 10.40; Cl, 12.62. Found: C, 77.21; H, 10.24; Cl, 12.15.

\section{Substitution Products (4, 10i, 16, and 20), ${ }^{13} \mathrm{C}$ NMR spectra, and NMR Assignments.}

2-Ethylidene-1,1,3,3-tetramethylindan (4d). ${ }^{13} \mathrm{C} \mathrm{NMR}\left(100.6 \mathrm{MHz}, \mathrm{CDCl}_{3}\right) \delta 14.09\left(\mathrm{C}^{\beta}\right), 29.35$ (2 1- $\left.\mathrm{CH}_{3}\right), 32.52\left(23-\mathrm{CH}_{3}\right), 46.37\left(\mathrm{C}^{1}\right), 47.10\left(\mathrm{C}^{3}\right), 116.24\left(\mathrm{C}^{\alpha}\right), 122.37$ and $122.58\left(\mathrm{C}^{4,7}\right), 126.80$ and $126.91\left(\mathrm{C}^{5,6}\right), 149.42\left(\mathrm{C}^{9}\right), 150.92\left(\mathrm{C}^{8}\right), 158.86\left(\mathrm{C}^{2}\right)$, assigned by selective $\left\{{ }^{1} \mathrm{H}\right\}$ decoupling in $\mathrm{THF}$ solution: $\left\{3-\mathrm{CH}_{3}\right\} \rightarrow \mathrm{C}^{3}\left(\mathrm{~d},{ }^{3} J=5.0 \mathrm{~Hz}\right)$ and $\mathrm{C}^{\alpha}\left(\mathrm{dq},{ }^{1} J=148.0 \mathrm{~Hz},{ }^{2} J=6.8 \mathrm{~Hz}\right)$ and $\mathrm{C}^{\beta}\left(\mathrm{dd},{ }^{1} J=123\right.$ $\left.\mathrm{Hz},{ }^{2} J=2.6 \mathrm{~Hz}\right)$ and $\mathrm{C}^{9}$ (pseudo-t), $\left\{1-\mathrm{CH}_{3}\right\} \rightarrow \mathrm{C}^{1}\left(\mathrm{~d},{ }^{3} J=9.4 \mathrm{~Hz}\right)$ and $\mathrm{C}^{\alpha}(\mathrm{dq})$ and $\mathrm{C}^{\beta}(\mathrm{dd})$ and $\mathrm{C}^{8}$ (dd). 
2-(1-Chloroethylidene)-1,1,3,3-tetramethylindan (4f). ${ }^{13} \mathrm{C} \mathrm{NMR}\left(100.6 \mathrm{MHz}, \mathrm{CDCl}_{3}\right) \quad \delta \quad 25.69$ $\left(\mathrm{C}^{\beta}\right), 28.03\left(21-\mathrm{CH}_{3}\right), 29.52\left(23-\mathrm{CH}_{3}\right), 48.38$ and $49.40\left(\mathrm{C}^{1,3}\right), 122.26\left(\mathrm{C}^{4}\right), 122.48\left(\mathrm{C}^{7}\right), 125.86\left(\mathrm{C}^{\alpha}\right)$, $127.13\left(C^{5}\right), 127.24\left(C^{6}\right), 149.45$ and $149.92\left(C^{8,9}\right), 152.09\left(C^{2}\right)$, assigned by ${ }^{1} \mathrm{H} /{ }^{13} \mathrm{C}$ heterocorrelation.

\section{Scheme 5}
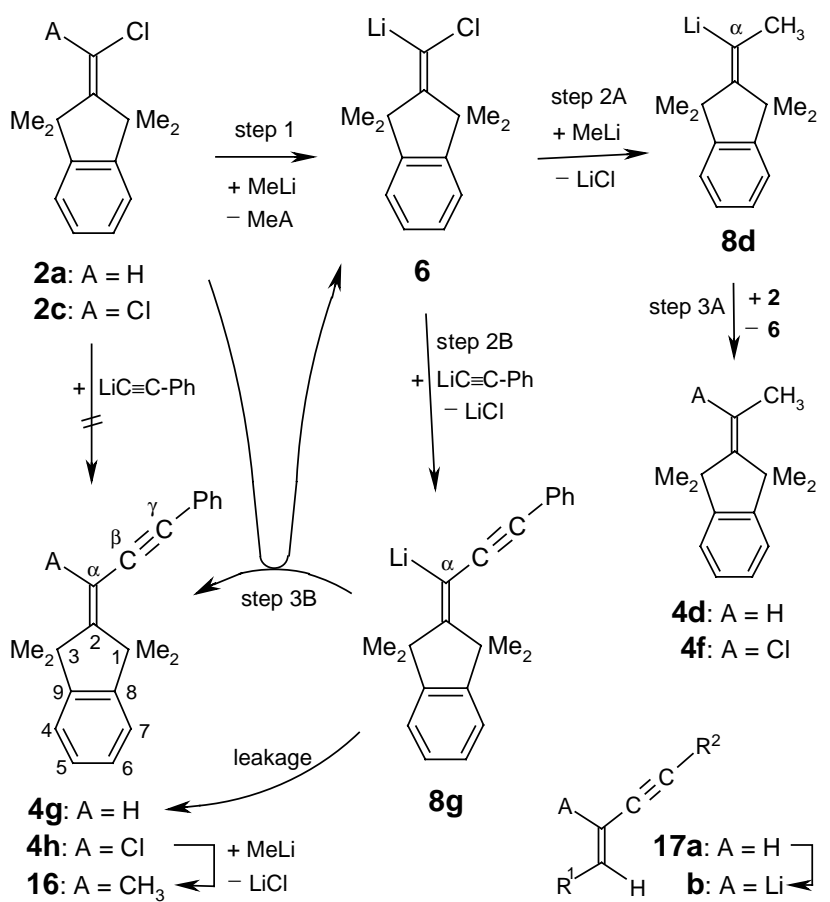

4d: $\mathrm{A}=\mathrm{H}$

4f: $\mathrm{A}=\mathrm{Cl}$

2-(3-Phenyl-2-propyn-1-ylidene)-1,1,3,3-tetramethylindan (4g). A dry NMR tube (5 mm) was charged under argon cover gas with MeLi $(0.57 \mathrm{mmol})$ in $\mathrm{Et}_{2} \mathrm{O}(0.46 \mathrm{~mL})$, anhydrous THF $(0.5 \mathrm{~mL})$, and $\left[\mathrm{D}_{12}\right]$-cyclohexane (NMR lock, $0.080 \mathrm{~mL}$ ), then cooled at $-78{ }^{\circ} \mathrm{C}$. After injection of phenylacetylene $(0.054 \mathrm{~mL}, 0.50 \mathrm{mmol})$, the solution contained phenylethynyllithium $(0.50 \mathrm{mmol})$ and residual MeLi (0.027 mmol), checked by ${ }^{1} \mathrm{H}$ NMR. Solid monochloride 2a (60 mg, $\left.0.27 \mathrm{mmol}\right)$ was added at $-30{ }^{\circ} \mathrm{C}$ and dissolved by vigorous shaking at room temperature, whereupon all MeLi was consumed within $10 \mathrm{~min}$. The next day, MeLi $(0.020 \mathrm{~mL}, 0.025 \mathrm{mmol})$ was injected, and the reaction was observed by ${ }^{1} \mathrm{H}$ NMR which revealed that MeLi disappeared again within 10 min while the product $4 \mathrm{~g}$ continued to increase over the next $140 \mathrm{~min}$. After another night at room temperature and the subsequent injection of more MeLi $(0.020 \mathrm{~mL}, 0.025 \mathrm{mmol}), \mathbf{2} \mathbf{a}$ and MeLi approached the detection limit in the course of five hours and vanished over the third night. The tube was emptied onto solid $\mathrm{CO}_{2}$, and the product mixture was dissolved in $\mathrm{Et}_{2} \mathrm{O}$ plus $2 \mathrm{M} \mathrm{NaOH}$. The acidified $\mathrm{NaOH}$ layer furnished phenylpropiolic acid only $(30 \mathrm{mg}, 89 \%)$, mp $136-138{ }^{\circ} \mathrm{C}\left(\mathrm{CCl}_{4}\right.$; ref S13: 136-137 $\left.{ }^{\circ} \mathrm{C}\right)$. The $\mathrm{Et}_{2} \mathrm{O}$ layer was washed until neutral and dried over $\mathrm{MgSO}_{4}$ to provide almost pure $\mathbf{4 g}(52 \mathrm{mg}, 67 \%$ ), which was distilled at $180-210{ }^{\circ} \mathrm{C}$ (bath temp.)/1 mbar and then crystallized from ethanol: colorless thin needles (34 mg), mp 82-83 ${ }^{\circ} \mathrm{C} ;{ }^{1} \mathrm{H}$ NMR (400 MHz, $\mathrm{CDCl}_{3}$, numbering of Scheme 5) $\delta 1.41(\mathrm{~s}$, 
2 3- $\mathrm{CH}_{3}$ ), 1.70 (s, 2 1- $\left.\mathrm{CH}_{3}\right), 5.83$ (s, $\left.\alpha-\mathrm{H}\right), 7.18$ (m, 4-H), 7.21 (m, 7-H), 7.25 (m, 5-/6-H), 7.32 (tm, 2 $m-\mathrm{H}), 7.34(\mathrm{~m}, p-\mathrm{H})$, and $7.47(\mathrm{dm}, 2 o-\mathrm{H})$, assigned by the NOESY correlations $\alpha-\mathrm{H} \leftrightarrow 3-\mathrm{CH}_{3} \leftrightarrow 4-$ $\mathrm{H}, 1-\mathrm{CH}_{3} \leftrightarrow 7-\mathrm{H}$, and $o-\mathrm{H} \leftrightarrow m-\mathrm{H} ;{ }^{13} \mathrm{C} \mathrm{NMR}\left(100.6 \mathrm{MHz}, \mathrm{CDCl}_{3}\right) \delta 28.55\left(\mathrm{qq},{ }^{1} J=127 \mathrm{~Hz},{ }^{3} J=4.6\right.$ $\left.\mathrm{Hz}, 21-\mathrm{CH}_{3}\right), 31.93\left(\mathrm{qq},{ }^{1} J=127 \mathrm{~Hz},{ }^{3} J=4.6 \mathrm{~Hz}, 23-\mathrm{CH}_{3}\right), 48.00\left(\mathrm{ddm},{ }^{3} J=8.0 \mathrm{~Hz},{ }^{3} J=2.0 \mathrm{~Hz}, \mathrm{C}^{1}\right)$, $48.09\left(\mathrm{ddm},{ }^{3} J=4.4 \mathrm{~Hz},{ }^{3} J=2 \mathrm{~Hz}, \mathrm{C}^{3}\right), 87.11\left(\right.$ narrow $\left.\mathrm{m}, \mathrm{C}^{\beta}\right), 96.16\left(\mathrm{td},{ }^{3} J=5 \mathrm{~Hz},{ }^{3} J=4.7 \mathrm{~Hz}, \mathrm{C}^{\gamma}\right)$, $101.22\left(\operatorname{sharp~d},{ }^{1} J=160 \mathrm{~Hz}, \mathrm{C}^{\alpha}\right), 122.53\left(\mathrm{dm},{ }^{1} J=156.8 \mathrm{~Hz}, \mathrm{C}^{4,7}\right), 124.07\left(\mathrm{t},{ }^{3} J=7.5 \mathrm{~Hz}, \mathrm{C}^{\mathrm{i}}\right), 127.17$ $\left(\mathrm{ddd},{ }^{1} J=159 \mathrm{~Hz},{ }^{3} J=7 \mathrm{~Hz}, \mathrm{C}^{5}\right), 127.34\left(\mathrm{ddd},{ }^{1} J=159 \mathrm{~Hz},{ }^{3} J=7 \mathrm{~Hz}, \mathrm{C}^{6}\right), 127.90\left(\mathrm{dt},{ }^{1} J=161 \mathrm{~Hz},{ }^{3} J\right.$ $\left.=7.5 \mathrm{~Hz}, \mathrm{C}^{p}\right), 128.37\left(\mathrm{dd},{ }^{1} J=160.5 \mathrm{~Hz},{ }^{3} J=7 \mathrm{~Hz}, \mathrm{C}^{m}\right), 130.91\left(\mathrm{dt},{ }^{1} J=162.4 \mathrm{~Hz},{ }^{3} J=6.3 \mathrm{~Hz}, \mathrm{C}^{o}\right)$, $148.14\left(\mathrm{tm},{ }^{3} J=6.3 \mathrm{~Hz}, \mathrm{C}^{9}\right), 149.73\left(\mathrm{tm},{ }^{3} J=6.3 \mathrm{~Hz}, \mathrm{C}^{8}\right), 174.92\left(\mathrm{~m},{ }^{3} J \approx 3.5 \mathrm{~Hz}, \mathrm{C}^{2}\right)$, assigned by ${ }^{1} \mathrm{H} /{ }^{13} \mathrm{C}$ heterocorrelation and by selective $\left\{{ }^{1} \mathrm{H}\right\}$ decoupling: $\left\{3-\mathrm{CH}_{3}\right\} \rightarrow \mathrm{C}^{3}(\mathrm{dd})$ and $\mathrm{C}^{9}(\mathrm{t}),\left\{1-\mathrm{CH}_{3}\right\} \rightarrow$ $\mathrm{C}^{1}(\mathrm{dd})$ and $\mathrm{C}^{8}(\mathrm{t}),\{\alpha-\mathrm{H}\} \rightarrow \mathrm{C}^{\gamma}(\mathrm{t}),\{$ ortho-H $\} \rightarrow \mathrm{C}^{\gamma}(\mathrm{d})$; IR $(\mathrm{KBr})$ 2961, $2200(\mathrm{w}), 1490,1444,754$ (s), and $690 \mathrm{~cm}^{-1}$. Anal. Calcd for $\mathrm{C}_{22} \mathrm{H}_{22}$ (286.4): C, 92.26; H, 7.74. Found: C, 91.74; H, 7.80.

\section{Scheme 7}

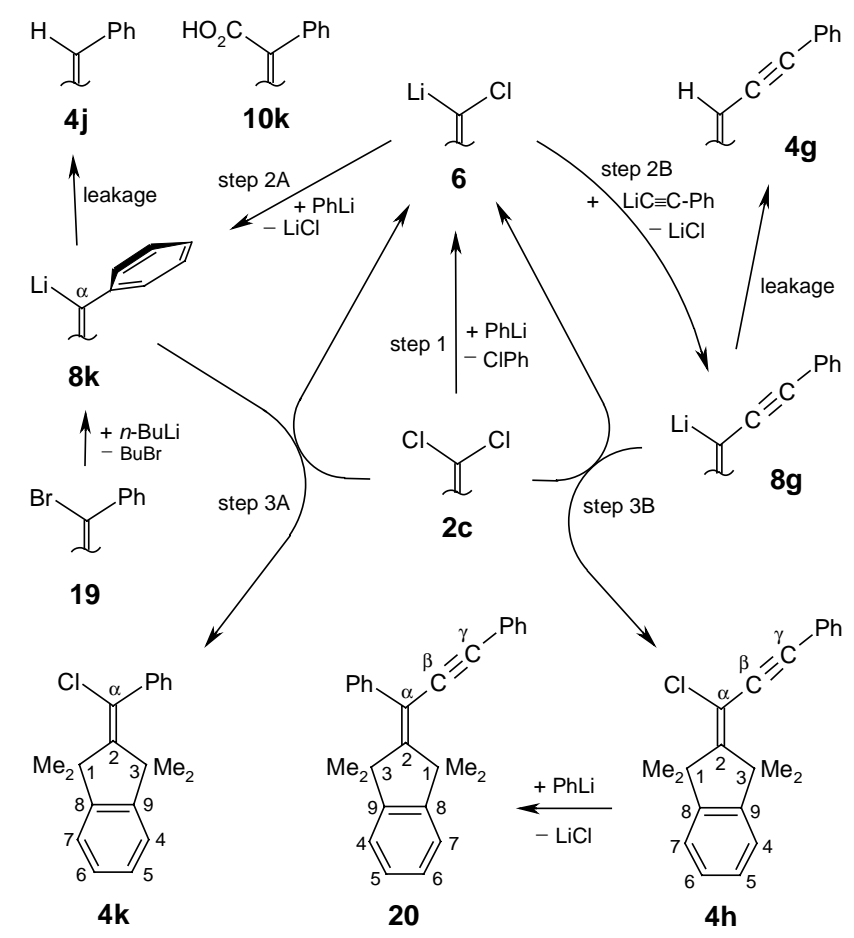

2-(1-Chloro-3-phenyl-2-propyn-1-ylidene)-1,1,3,3-tetramethylindan (4h). A solution of LiMes* (21p, 0.2 equiv) in $\mathrm{Et}_{2} \mathrm{O}(0.25 \mathrm{~mL})$ was added under argon cover gas to $\mathrm{PhC} \equiv \mathrm{CLi}(0.58 \mathrm{mmol}, 1.5$ equiv) in THF $(0.4 \mathrm{~mL})$ in a dried NMR tube kept at $-78{ }^{\circ} \mathrm{C}$. After the introduction of dichloride $2 \mathbf{c}$ (100 mg, $0.39 \mathrm{mmol}$ ), the tube was sealed and warmed up with shaking at room temperature. The next day, another batch of LiMes* $\left(0.1\right.$ equiv) in $\mathrm{Et}_{2} \mathrm{O}(0.2 \mathrm{~mL})$ was added. After two further nights, the 
mixture was poured onto solid $\mathrm{CO}_{2}$, warmed up, and dissolved in $2 \mathrm{M} \mathrm{NaOH}$ and $\mathrm{Et}_{2} \mathrm{O}$. The washed $\left(\mathrm{Et}_{2} \mathrm{O}\right)$ and acidified $\mathrm{NaOH}$ phase furnished $23 \mathrm{mg}(0.16 \mathrm{mmol})$ of $\mathrm{PhC} \equiv \mathrm{CCO}_{2} \mathrm{H}$, indicating that 0.41 equiv of $\mathrm{PhC} \equiv \mathrm{CLi}$ had survived. The combined and washed $\mathrm{Et}_{2} \mathrm{O}$ layers afforded a mixture (169 mg) of 2c, $\mathbf{4 g}, \mathbf{4 h}, \mathrm{ClMes}^{*}$, and HMes* in the molar ratio 18:8:35:21:18. Elution of the contaminations by chromatography on silica (4.0 g) with petroleum ether (low-boiling) provided $\mathbf{4 h}$ as a yellow oil that crystallized from methanol: mp $109-110.5{ }^{\circ} \mathrm{C}\left(\mathrm{CH}_{3} \mathrm{OH}\right) ;{ }^{1} \mathrm{H}$ NMR $\left(400 \mathrm{MHz}, \mathrm{CDCl}_{3}\right) \delta 1.66$ and $1.70\left(2 \mathrm{~s}, 2+21-/ 3-\mathrm{CH}_{3}\right), 7.17$ and $7.26(2 \mathrm{~m}, 2+2$ indan-H), $7.37(\mathrm{~m}, 2 \mathrm{~m}-\mathrm{H}$ and $1 p-\mathrm{H}), 7.52$ (m, 2 $o-\mathrm{H}) ;{ }^{13} \mathrm{C} \mathrm{NMR}\left(100.6 \mathrm{MHz}, \mathrm{CDCl}_{3}\right.$, numbering of Scheme 7) $\delta 27.43$ and $28.44\left(2+21-/ 3-\mathrm{CH}_{3}\right)$, 49.68 and $50.24\left(\mathrm{C}^{1,3}\right), 86.90\left(\mathrm{C}^{\beta}\right), 94.74\left(\mathrm{C}^{\gamma}\right), 108.98\left(\mathrm{C}^{\alpha}\right), 122.38\left(\mathrm{C}^{4,7}\right), 122.57\left(\mathrm{C}^{\mathrm{i}}\right), 127.46$ and $127.48\left(\mathrm{C}^{5,6}\right), 128.49\left(2 \mathrm{C}^{m}\right), 128.89\left(\mathrm{C}^{p}\right), 131.22\left(2 \mathrm{C}^{o}\right), 148.72$ and $149.01\left(\mathrm{C}^{8,9}\right), 165.12\left(\mathrm{C}^{2}\right)$, assigned by comparison with 4g; IR (KBr) 2964, 2927, $2202(\mathrm{w}), 1486,848(\mathrm{C}-\mathrm{Cl}), 756,688 \mathrm{~cm}^{-1}$. Anal. Calcd for $\mathrm{C}_{22} \mathrm{H}_{21} \mathrm{Cl}$ (320.9): C, 82.35; H, 6.60; Cl, 11.05. Found: C, 82.28; H, 6.56; $\mathrm{Cl}$, 11.26.

2-( $\alpha$-Chlorobenzylidene)-1,1,3,3-tetramethylindan (4k). Crystalline 8k (Scheme 7) was prepared ${ }^{46}$ from the bromoalkene 19 with $n$-BuLi, washed with pentane $(4 \times)$ to remove the self-coupled allenic

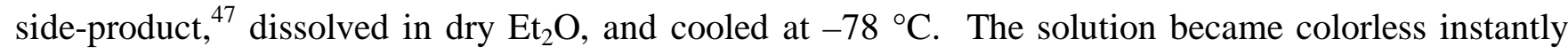
upon the addition of hexachloroethane $(173 \mathrm{mg}, 0.73 \mathrm{mmol})$. After $1 \mathrm{~h}$ at room temperature and addition of water, the mixture was extracted with $\mathrm{Et}_{2} \mathrm{O}(3 \times)$, and the combined $\mathrm{Et}_{2} \mathrm{O}$ layers were washed until neutral and dried over $\mathrm{MgSO}_{4}$. The filtered and concentrated material crystallized from methanol $(2 \times)$ as colorless needles of pure $4 \mathbf{k}(141 \mathrm{mg}, 65 \%)$ : $\mathrm{mp} 108-110{ }^{\circ} \mathrm{C} ;{ }^{1} \mathrm{H} \mathrm{NMR}(400 \mathrm{MHz}$, $\left.\mathrm{CDCl}_{3}\right) \delta 1.18\left(\mathrm{~s}, 23-\mathrm{CH}_{3}\right), 1.76\left(\mathrm{~s}, 21-\mathrm{CH}_{3}\right), 7.03(\mathrm{~m}, 4-\mathrm{H}), 7.20(\mathrm{~m}, 7-\mathrm{H}), 7.21(\mathrm{~m}, 5-\mathrm{H}), 7.25(\mathrm{~m}$, 6-H), $7.34(\mathrm{~m}, p-\mathrm{H}), 7.36(\mathrm{~m}, 2 o-\mathrm{H})$, and $7.38(\mathrm{~m}, 2 \mathrm{~m}-\mathrm{H})$, assigned by the NOESY correlations ortho$\mathrm{H} \leftrightarrow 3-\mathrm{CH}_{3} \leftrightarrow 4-\mathrm{H} \leftrightarrow 5-\mathrm{H}$ and $1-\mathrm{CH}_{3} \leftrightarrow 7-\mathrm{H} \leftrightarrow 6-\mathrm{H} ;{ }^{13} \mathrm{C} \mathrm{NMR}\left(100.6 \mathrm{MHz}, \mathrm{CDCl}_{3}\right) \delta 27.97$ (qq, $\left.{ }^{1} J=127 \mathrm{~Hz},{ }^{3} J=4.7 \mathrm{~Hz}, 21-\mathrm{CH}_{3}\right), 31.16\left(\mathrm{qq},{ }^{1} J=127 \mathrm{~Hz},{ }^{3} J=4.7 \mathrm{~Hz}, 23-\mathrm{CH}_{3}\right), 49.04\left(\mathrm{dm},{ }^{3} J=1.8\right.$ $\left.\mathrm{Hz}, \mathrm{C}^{3}\right), 49.92\left(\mathrm{dm},{ }^{3} J=1.5 \mathrm{~Hz}, \mathrm{C}^{1}\right), 122.17\left(\mathrm{ddd},{ }^{1} J=157 \mathrm{~Hz},{ }^{3} J=7.8 \mathrm{~Hz}, \mathrm{C}^{4}\right), 122.44\left(\mathrm{ddm},{ }^{1} J=157\right.$ $\left.\mathrm{Hz},{ }^{3} J=7.8 \mathrm{~Hz}, \mathrm{C}^{7}\right), 127.16\left(\mathrm{ddm},{ }^{1} J=159 \mathrm{~Hz}, \mathrm{C}^{5}\right), 127.25\left(\mathrm{ddd},{ }^{1} J=159 \mathrm{~Hz}, \mathrm{C}^{6}\right), 127.61\left(\mathrm{br} \mathrm{t},{ }^{3} J \approx 3\right.$ $\left.\mathrm{Hz}, \mathrm{C}^{\alpha}\right), 127.95\left(\mathrm{ddm},{ }^{1} J=160 \mathrm{~Hz}, 2 \mathrm{C}^{m}\right), 128.06\left(\mathrm{dtt},{ }^{1} J=160 \mathrm{~Hz},{ }^{3} J=6.7 \mathrm{~Hz}, \mathrm{C}^{p}\right), 130.01\left(\mathrm{dtm},{ }^{1} J=\right.$ $\left.160 \mathrm{~Hz}, 2 \mathrm{C}^{o}\right), 140.56\left(\mathrm{t},{ }^{3} J=6.8 \mathrm{~Hz}, \mathrm{C}^{i}\right), 149.07\left(\mathrm{tm},{ }^{3} J=6.7 \mathrm{~Hz}, \mathrm{C}^{9}\right), 149.65\left(\mathrm{tm},{ }^{3} J=6.7 \mathrm{~Hz}, \mathrm{C}^{8}\right)$, $155.13\left(\mathrm{~m},{ }^{3} \mathrm{~J} \approx 3.5 \mathrm{~Hz}, \mathrm{C}^{2}\right)$, assigned by ${ }^{1} \mathrm{H} /{ }^{13} \mathrm{C}$ heterocorrelation and by selective $\left\{{ }^{1} \mathrm{H}\right\}$ decoupling: $\{3$ $\left.\mathrm{CH}_{3}\right\} \rightarrow \mathrm{C}^{3}(\mathrm{~d})$ and $\mathrm{C}^{9}$ (pseudo-t), $\left\{1-\mathrm{CH}_{3}\right\} \rightarrow \mathrm{C}^{1}(\mathrm{~d})$ and $\mathrm{C}^{8}(\mathrm{t}) ; \mathrm{IR}(\mathrm{KBr})$ 2990, 2962, $1648(\mathrm{w})$, 1484, 839, 759 (vs), 716, and $701 \mathrm{~cm}^{-1}$. Anal. Calcd for $\mathrm{C}_{20} \mathrm{H}_{21} \mathrm{Cl}$ (296.8): C, 80.93; H, 7.13; Cl, 11.94. Found: C, 81.06; H, 7.26; Cl, 11.21 . 
2-(4-Trimethylsilylbenzylidene)-1,1,3,3-tetramethylindan (4l). $n$-BuLi (2.4 mmol) in hexane (1.0 $\mathrm{mL})$ was added with stirring to the chloroalkene $\mathbf{4 m}(339 \mathrm{mg}, 0.92 \mathrm{mmol}$, Scheme 8$)$ in $t$-BuOMe (5.0 $\mathrm{mL})$ and stirred for one day at room temperature. The mixture was worked up with water and $\mathrm{Et}_{2} \mathrm{O}(3 \times$ $3 \mathrm{~mL}$ ). The combined $\mathrm{Et}_{2} \mathrm{O}$ phases were washed until neutral, dried over $\mathrm{MgSO}_{4}$, evaporated to dryness, and distilled at $113-133{ }^{\circ} \mathrm{C}$ (bath temperature) $/ 10^{-3}$ mbar to give $133 \mathrm{mg}(43 \%)$ of almost pure 4l: $\mathrm{mp}$ 63.5-65 ${ }^{\circ} \mathrm{C}$ (methanol); ${ }^{1} \mathrm{H} \mathrm{NMR}\left(400 \mathrm{MHz}, \mathrm{CDCl}_{3}\right) \delta 0.28\left(\mathrm{~s}, \mathrm{SiMe}_{3}\right), 1.31\left(\mathrm{~s}, 2\right.$ 1- $\left.\mathrm{CH}_{3}\right)$, 1.48 (s, 2 3- $\left.\mathrm{CH}_{3}\right), 6.63$ (s, $\left.\alpha-\mathrm{H}\right), 7.11(\mathrm{dm}, 7-\mathrm{H}), 7.18-7.23(\mathrm{~m}, 4-/ 5-/ 6-\mathrm{H}), 7.25\left(\mathrm{dm},{ }^{3} \mathrm{~J}=8 \mathrm{~Hz}, 2 o-\mathrm{H}\right)$, and $7.47\left(\mathrm{dt},{ }^{3} \mathrm{~J}=8 \mathrm{~Hz}, 2 \mathrm{~m}-\mathrm{H}\right)$, assigned by the NOE difference correlations ortho- $\mathrm{H} \leftarrow\left\{1-\mathrm{CH}_{3}\right\} \rightarrow 7$ $\mathrm{H}$ and $\alpha-\mathrm{H} \leftarrow\left\{3-\mathrm{CH}_{3}\right\} \rightarrow 4-\mathrm{H} ;{ }^{13} \mathrm{C} \mathrm{NMR}\left(100.6 \mathrm{MHz}, \mathrm{CDCl}_{3}\right) \delta-1.0\left(\mathrm{qm},{ }^{1} J=119 \mathrm{~Hz},{ }^{3} J=2 \mathrm{~Hz}\right.$, $\left.{ }^{1} J\left({ }^{13} \mathrm{C}^{29} \mathrm{Si}\right)=52 \mathrm{~Hz}, \mathrm{SiMe}_{3}\right), 31.00\left(\mathrm{qq},{ }^{1} J=127 \mathrm{~Hz},{ }^{3} J=4.6 \mathrm{~Hz}, 21-\mathrm{CH}_{3}\right), 32.4\left(\mathrm{qq},{ }^{1} J=127 \mathrm{~Hz},{ }^{3} J=\right.$ $\left.4.6 \mathrm{~Hz}, 23-\mathrm{CH}_{3}\right), 47.2\left(\mathrm{ddm},{ }^{3} J=9.0 \mathrm{~Hz},{ }^{3} J=2 \mathrm{~Hz}, \mathrm{C}^{1}\right), 47.8\left(\mathrm{ddm},{ }^{3} J=4.8 \mathrm{~Hz},{ }^{3} J=2 \mathrm{~Hz}, \mathrm{C}^{3}\right), 122.3$ $\left(\mathrm{dm},{ }^{1} J=156 \mathrm{~Hz}, \mathrm{C}^{7}\right), 122.5\left(\mathrm{dm},{ }^{1} J=156 \mathrm{~Hz}, \mathrm{C}^{4}\right), 122.7\left(\mathrm{dt},{ }^{1} J=150.0 \mathrm{~Hz},{ }^{3} J=4.2 \mathrm{~Hz}, \mathrm{C}^{\alpha}\right), 126.9$ $\left(\mathrm{ddm},{ }^{1} J=159 \mathrm{~Hz}, \mathrm{C}^{5}\right), 127.1\left(\mathrm{ddm},{ }^{1} J=159 \mathrm{~Hz}, \mathrm{C}^{6}\right), 128.7\left(\mathrm{dm},{ }^{1} J=156 \mathrm{~Hz}, 2 \mathrm{C}^{o}\right), 132.7\left(\mathrm{ddm},{ }^{1} J=\right.$ $155 \mathrm{~Hz}, 2 \mathrm{C}^{m}$ ), 137.9 (unresolved, $\left.\mathrm{C}^{p}\right), 139.4\left(\mathrm{t},{ }^{3} J=7.4 \mathrm{~Hz}, \mathrm{C}^{i}\right), 148.7\left(\mathrm{tm}, \mathrm{C}^{9}\right), 150.6\left(\mathrm{tm}, \mathrm{C}^{8}\right), 161.9$ $\left(\mathrm{m},{ }^{3} \mathrm{~J} \approx 3.5 \mathrm{~Hz}, \mathrm{C}^{2}\right)$, assigned by ${ }^{1} \mathrm{H} /{ }^{13} \mathrm{C}$ heterocorrelation and by selective $\left\{{ }^{1} \mathrm{H}\right\}$ decoupling: $\left\{1-\mathrm{CH}_{3}\right\}$ $\rightarrow \mathrm{C}^{1}(\mathrm{dd})$ and $\mathrm{C}^{8}$ (pseudo-t), $\left\{3-\mathrm{CH}_{3}\right\} \rightarrow \mathrm{C}^{3}(\mathrm{dd})$ and $\mathrm{C}^{9}(\mathrm{dd}),\{7-\mathrm{H}\} \rightarrow \mathrm{C}^{7}(\mathrm{~s}),\{m-\mathrm{H}\} \rightarrow \mathrm{C}^{m}(\mathrm{~s})$ and $\mathrm{C}^{i}(\mathrm{~s})$. IR (KBr) 2957, $1596(\mathrm{vw}), 1483(\mathrm{w}), 1250(\mathrm{w}), 1108,840(\mathrm{~s})$, and $758 \mathrm{~cm}^{-1}$. Anal. Calcd for $\mathrm{C}_{23} \mathrm{H}_{30} \mathrm{Si}$ (334.6): C, 82.57; H, 9.04. Found: C, 82.29; H, 9.17.

\section{Scheme 8}
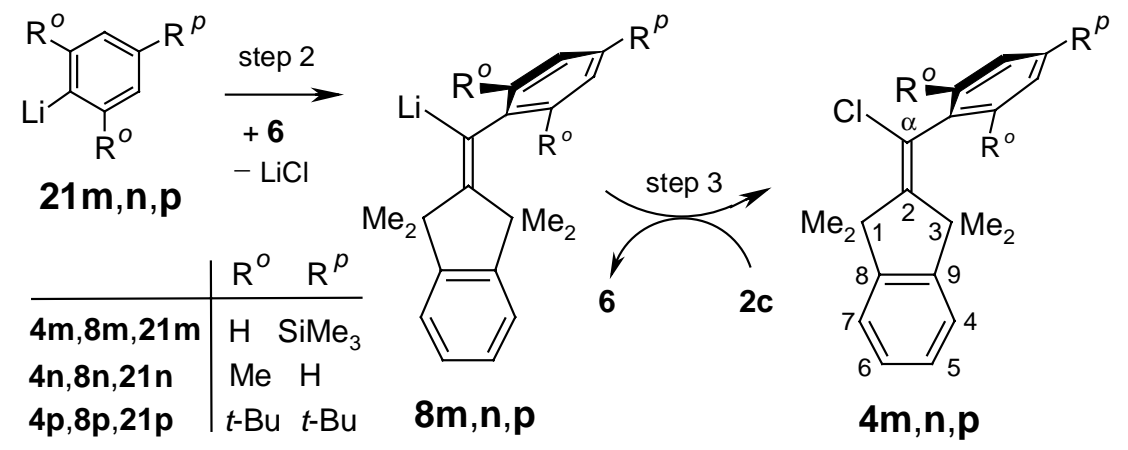

2-( $\alpha$-Chloro-4-trimethylsilylbenzylidene)-1,1,3,3-tetramethylindan (4m). ${ }^{13} \mathrm{C}$ NMR (100.6 MHz, $\left.\mathrm{CDCl}_{3}\right) \quad \delta-1.1\left(\mathrm{qm},{ }^{1} J=119.5 \mathrm{~Hz},{ }^{3} J=2 \mathrm{~Hz},{ }^{1} J\left({ }^{13} \mathrm{C}^{29} \mathrm{Si}\right)=52.3 \mathrm{~Hz}, \mathrm{SiMe}_{3}\right), 28.0\left(\mathrm{qq},{ }^{1} J=127.5 \mathrm{~Hz}\right.$, $\left.{ }^{3} J=4.5 \mathrm{~Hz}, 21-\mathrm{CH}_{3}\right), 31.2\left(\mathrm{qq},{ }^{1} J=127.5 \mathrm{~Hz},{ }^{3} J=4.5 \mathrm{~Hz}, 23-\mathrm{CH}_{3}\right), 49.1\left(\mathrm{~m}, \mathrm{C}^{3}\right), 49.9\left(\mathrm{~m}, \mathrm{C}^{1}\right), 122.2$ $\left(\mathrm{ddm},{ }^{1} J=157 \mathrm{~Hz}, \mathrm{C}^{4}\right), 122.4\left(\mathrm{ddm},{ }^{1} J=157 \mathrm{~Hz}, \mathrm{C}^{7}\right), 127.1\left(\mathrm{ddm},{ }^{1} J=160 \mathrm{~Hz}, \mathrm{C}^{5}\right), 127.2\left(\mathrm{ddm},{ }^{1} J=\right.$ $\left.160 \mathrm{~Hz}, \mathrm{C}^{6}\right), 127.6\left(\mathrm{t},{ }^{3} J \approx 5 \mathrm{~Hz}, \mathrm{C}^{\alpha}\right), 129.1\left(\mathrm{dm},{ }^{1} J \approx 158 \mathrm{~Hz}, 2 \mathrm{C}^{o}\right), 132.9\left(\mathrm{dm},{ }^{1} J \approx 158 \mathrm{~Hz}, 2 \mathrm{C}^{m}\right)$, 
$140.5\left(\mathrm{~m}, \mathrm{C}^{p}\right), 140.8\left(\mathrm{t},{ }^{3} J=7.6 \mathrm{~Hz}, \mathrm{C}^{i}\right), 149.1\left(\mathrm{~m}, \mathrm{C}^{9}\right), 149.7\left(\mathrm{~m}, \mathrm{C}^{8}\right), 155.1\left(\mathrm{~m}, \mathrm{C}^{2}\right)$, assigned by comparison with $\mathbf{4 k}$.

2-( $\alpha$-Chloro-2,6-dimethylbenzylidene)-1,1,3,3-tetramethylindan (4n). ${ }^{13} \mathrm{C} \mathrm{NMR}(100.6 \mathrm{MHz}$, $\left.\mathrm{CDCl}_{3}\right) \delta 20.1\left(\mathrm{qd},{ }^{1} J=127 \mathrm{~Hz},{ }^{3} J=5 \mathrm{~Hz}, 2 o-\mathrm{CH}_{3}\right), 27.9\left(\mathrm{qq},{ }^{1} J=127.7 \mathrm{~Hz},{ }^{3} J=4.5 \mathrm{~Hz}, 21-\mathrm{CH}_{3}\right)$, $28.8\left(\mathrm{qq},{ }^{1} J=127.7 \mathrm{~Hz},{ }^{3} J=4.5 \mathrm{~Hz}, 23-\mathrm{CH}_{3}\right), 49.4\left(\mathrm{~m}, \mathrm{C}^{1}\right), 50.1\left(\mathrm{~m}, \mathrm{C}^{3}\right), 122.1\left(\mathrm{dd},{ }^{1} J=158 \mathrm{~Hz},{ }^{3} J=\right.$ $\left.7.5 \mathrm{~Hz}, \mathrm{C}^{4}\right), 122.4\left(\mathrm{dd},{ }^{1} \mathrm{~J}=157 \mathrm{~Hz},{ }^{3} \mathrm{~J}=7.5 \mathrm{~Hz}, \mathrm{C}^{7}\right), 126.0\left(\operatorname{sharp~s}, \mathrm{C}^{\alpha}\right), 127.1\left(\mathrm{ddm},{ }^{1} \mathrm{~J} \approx 160 \mathrm{~Hz},{ }^{3} \mathrm{~J}=\right.$ $\left.7 \mathrm{~Hz}, \mathrm{C}^{6}\right), 127.3\left(\mathrm{ddm},{ }^{1} J \approx 159 \mathrm{~Hz},{ }^{3} J=7 \mathrm{~Hz}, \mathrm{C}^{5}\right), 127.4\left(\mathrm{ddq},{ }^{1} J=159 \mathrm{~Hz}, 2 \mathrm{C}^{m}\right), 128.3\left(\operatorname{sharp~d},{ }^{1} J=\right.$ $\left.159.5 \mathrm{~Hz}, \mathrm{C}^{p}\right), 136.8\left(\mathrm{dq},{ }^{2} J \approx{ }^{3} J \approx 6.5 \mathrm{~Hz}, 2 \mathrm{C}^{o}\right), 138.9\left(\mathrm{br} \mathrm{m}, \mathrm{C}^{i}\right), 149.0\left(\mathrm{~m}, \mathrm{C}^{8}\right), 149.7\left(\mathrm{~m}, \mathrm{C}^{9}\right), 153.3$ $\left(\mathrm{m}, \mathrm{C}^{2}\right)$, assigned by comparisons with $\mathbf{1 9}^{\mathrm{S} 14}$ and $\mathbf{4 0}$.

\section{Scheme S1}

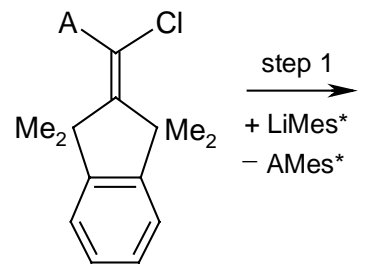

2a: $A=H$

2b: $A=D$

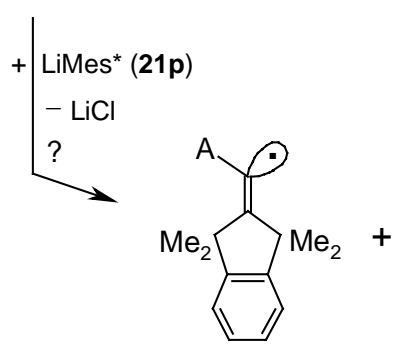

7

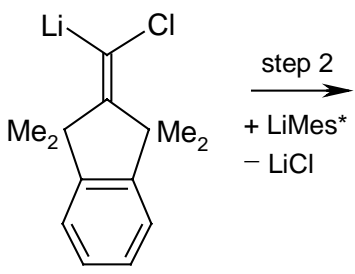

6

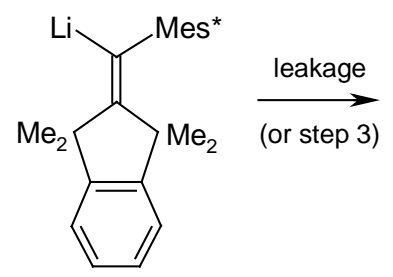

$8 p$

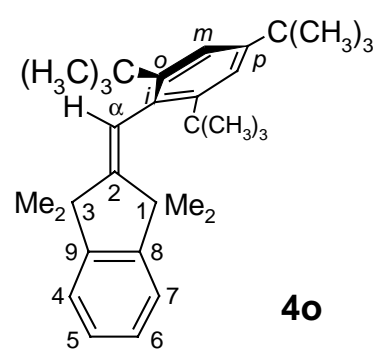

40

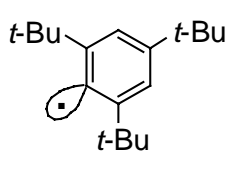

S1

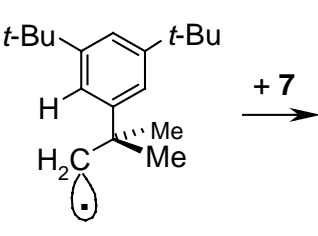

S2

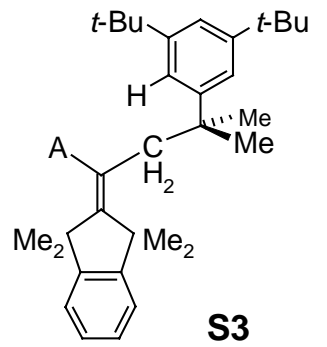

2-(2,4,6-Tri-tert-butylbenzylidene)-1,1,3,3-tetramethylindan (4o). The deprotonation of reagent $2 \mathbf{a}(0.10 \mathrm{mmol}, 0.19 \mathrm{M}$ in THF, Scheme S1) with an excess of LiMes* (see 4p below) took place with a first $t_{1 / 2} \approx 20 \mathrm{~min},{ }^{16}$ furnishing $\mathbf{4 o}^{52}$ (merely $0.021 \mathrm{mmol}$ ), HMes*, and unknown side-products. The intermediacy of carbenoid $\mathbf{6}$ was established under the same conditions with reagent $\mathbf{2 b}$ which reacted much more slowly (at least tenfold) ${ }^{16}$ to afford plenty of ethylene (from THF with LiMes*) and, through a carboxylative workup 28 hours later, $\mathrm{HO}_{2} \mathrm{C}-\mathrm{Mes} *(0.13 \mathrm{mmol})$ and the totally unlabeled leakage product 40 (again merely $0.021 \mathrm{mmol}$ ). Such a loss of deuterium revealed that chain propagation (step 3) was no longer achieved here (non-chain carbenoid mechanism due to the faster leakage reaction) and excluded the possibility that this strongly retarded conversion of $\mathbf{2} \mathbf{b}$ might have proceeded by electron transfer from LiMes* to $\mathbf{2 c}$, which would have generated the aryl radical S1 
which can rearrange $\mathrm{e}^{\mathrm{S} 15}$ by internal hydrogen transfer with formation of $\mathbf{S 2}$. Indeed, products such as $\mathbf{S 3}$ bearing the $\mathrm{CH}_{2}$ group of $\mathbf{S 2}$ were not detected by ${ }^{13} \mathrm{C}$ NMR in the present studies.

${ }^{1} \mathrm{H}$ NMR (400 MHz, $\mathrm{CDCl}_{3}$, numbering of Scheme S1) $\delta 0.93$ (s, 2 1- $\left.\mathrm{CH}_{3}\right), 1.33$ (s, $\left.p-t-\mathrm{Bu}\right), 1.36(\mathrm{~s}$, $2 o-t-\mathrm{Bu}), 1.55\left(\mathrm{~s}, 23-\mathrm{CH}_{3}\right), 6.98\left(\mathrm{dm},{ }^{3} \mathrm{~J} \approx 8 \mathrm{~Hz}, 7-\mathrm{H}\right), 7.11(\mathrm{~s}, \alpha-\mathrm{H}), 7.16(\mathrm{~m}, 5-\mathrm{H}), 7.18(\mathrm{~m}, 4-\mathrm{H}$ and 6-H), $7.26(\mathrm{~s}, 2 \mathrm{~m}-\mathrm{H})$, as in ref 52; ${ }^{13} \mathrm{C} \mathrm{NMR}\left(100.6 \mathrm{MHz}, \mathrm{CDCl}_{3}\right) \delta 30.62\left(\mathrm{qq},{ }^{1} \mathrm{~J}=126 \mathrm{~Hz},{ }^{3} \mathrm{~J}=4.8\right.$ $\left.\mathrm{Hz}, 21-\mathrm{CH}_{3}\right), 31.29\left(\mathrm{qq},{ }^{1} \mathrm{~J}=126 \mathrm{~Hz},{ }^{3} \mathrm{~J}=4.8 \mathrm{~Hz}, 23-\mathrm{CH}_{3}\right), 31.52\left(\mathrm{qsept},{ }^{1} J=125 \mathrm{~Hz},{ }^{3} \mathrm{~J}=4.8 \mathrm{~Hz}, p-\right.$ $\mathrm{CMe}_{3}$ ), 33.85 (qsept, ${ }^{1} J=125.5 \mathrm{~Hz},{ }^{3} J=4.9 \mathrm{~Hz}, 2 o-\mathrm{CMe}_{3}$ ), 34.58 (partially hidden $\mathrm{m}, p-C \mathrm{Me}_{3}$ ), 38.18 $\left(\mathrm{m}, 2 o-\mathrm{CMe}_{3}\right), 46.73\left(\mathrm{~m}, \mathrm{C}^{1}\right), 48.02$ (narrower $\left.\mathrm{m}, \mathrm{C}^{3}\right), 121.17\left(\mathrm{dd},{ }^{1} J=152 \mathrm{~Hz},{ }^{3} J=7 \mathrm{~Hz}, 2 \mathrm{C}^{m}\right)$, $121.92\left(\mathrm{ddm},{ }^{1} J=157 \mathrm{~Hz},{ }^{3} J \approx 7 \mathrm{~Hz}, \mathrm{C}^{7}\right), 122.37\left(\mathrm{ddm},{ }^{1} J=155 \mathrm{~Hz}, \mathrm{C}^{4}\right), 125.58\left(\operatorname{sharp~d},{ }^{1} J=150.3\right.$ $\left.\mathrm{Hz}, \mathrm{C}^{\alpha}\right), 126.62\left(\mathrm{ddm},{ }^{1} J=159 \mathrm{~Hz},{ }^{3} J=7.6 \mathrm{~Hz}, \mathrm{C}^{5}\right), 126.92\left(\mathrm{ddm},{ }^{1} J=160 \mathrm{~Hz}, \mathrm{C}^{6}\right), 131.48\left(\mathrm{t},{ }^{3} J=6.9\right.$ $\left.\mathrm{Hz}, \mathrm{C}^{i}\right), 147.51\left(\mathrm{~m},{ }^{3} J \approx 3.5 \mathrm{~Hz}, \mathrm{C}^{p}\right), 148.56\left(\mathrm{~m},{ }^{3} J=3.5 \mathrm{~Hz}, 2 \mathrm{C}^{o}\right), 148.78\left(\mathrm{~m}, \mathrm{C}^{9}\right), 150.82\left(\mathrm{br} \mathrm{m}, \mathrm{C}^{8}\right)$, 152.69 (narrower $\mathrm{m}, \mathrm{C}^{2}$ ).

40 is identical with a known ${ }^{52}$ substance whose NMR spectra were incompletely reported (2 ortho-C missing) and not assigned. Our NMR assignments were achieved as follows: NOESY $\left({ }^{1} \mathrm{H},{ }^{1} \mathrm{H}\right)$ showed the correlations $o-t$ - $\mathrm{Bu} \leftrightarrow m-\mathrm{H} \leftrightarrow p$ - $t$ - $\mathrm{Bu}, o-t-\mathrm{Bu} \leftrightarrow 1-\mathrm{CH}_{3} \leftrightarrow 7-\mathrm{H} \leftrightarrow 6-\mathrm{H}, o-t-\mathrm{Bu} \leftrightarrow 3-\mathrm{CH}_{3} \leftrightarrow 4-\mathrm{H}$, $o-t-\mathrm{Bu} \leftrightarrow \alpha-\mathrm{H} \leftrightarrow 3-\mathrm{CH}_{3} .{ }^{13} \mathrm{C}$ NMR resonances of all $\mathrm{CH}$ and $\mathrm{CH}_{3}$ groups were assigned by ${ }^{1} \mathrm{H} /{ }^{13} \mathrm{C}$ heterocorrelation and confirmed by COLOCS $\left({ }^{13} \mathrm{C},{ }^{1} \mathrm{H}\right.$ with window $\left.5.7 \mathrm{~Hz}\right)$, which identified also the signals of carbon atoms carrying no hydrogen by the ${ }^{2} J_{\mathrm{CH}}$ correlations $\mathrm{C}^{1} \leftrightarrow 1-\mathrm{CH}_{3}, \mathrm{C}^{3} \leftrightarrow 3-\mathrm{CH}_{3}, o-$ $C \mathrm{Me}_{3} \leftrightarrow o-\mathrm{CMe}_{3}$, and $p-C \mathrm{Me}_{3} \leftrightarrow p-\mathrm{CMe}_{3}$, in addition to the ${ }^{3} J_{\mathrm{CH}}$ correlations $\mathrm{C}^{5} \leftrightarrow 7-\mathrm{H}, \mathrm{C}^{6} \leftrightarrow 4-\mathrm{H}$, $\mathrm{C}^{7} \leftrightarrow 5-\mathrm{H}, \mathrm{C}^{9} \leftrightarrow 3-\mathrm{CH}_{3} \leftrightarrow \mathrm{C}^{2} \leftrightarrow 1-\mathrm{CH}_{3} \leftrightarrow \mathrm{C}^{8} \leftrightarrow 6-\mathrm{H}, \mathrm{C}^{1} \leftrightarrow \alpha-\mathrm{H} \leftrightarrow \mathrm{C}^{3}, \alpha-\mathrm{H} \leftrightarrow \mathrm{C}^{o} \leftrightarrow o-\mathrm{CMe}_{3}, \mathrm{C}^{p}$ $\leftrightarrow p-\mathrm{CMe}_{3}, \mathrm{C}^{i} \leftrightarrow m-\mathrm{H} \leftrightarrow \mathrm{C}^{m}$, and $o-C \mathrm{Me}_{3} \leftrightarrow m-\mathrm{H} \leftrightarrow p-C \mathrm{Me}_{3}$.

2-( $\alpha$-Chloro-2,4,6-tri-tert-butylbenzylidene)-1,1,3,3-tetramethylindan (4p). The solution of bromo-2,4,6-tri-tert-butylbenzene (BrMes*, $1.20 \mathrm{~g}, 3.68 \mathrm{mmol})$ in THF $(1.5 \mathrm{~mL})$ and pentane $(6 \mathrm{~mL})^{\mathrm{S} 1}$ was placed in an oven-dried Schlenk flask $(25 \mathrm{~mL}$, with magnetic stirring bar) under dry argon and cooled to $-70{ }^{\circ} \mathrm{C}$. A hexane solution $(2.40 \mathrm{~mL})$ of $n$-BuLi $(5.02 \mathrm{mmol})$ was added dropwise with gentle swirling, which caused the immediate precipitation of 2,4,6-tri-tert-butylphenyllithium (LiMes*, 21p in Scheme 8). ${ }^{43}$ The cooling bath was removed and the Schlenk flask left at ambient temperature for 20 min, whereupon the supernatant liquid was withdrawn from the precipitate with a pipet under argon and discarded (although it contained some LiMes*). The colorless precipitate (LiMes*) was covered with pentane $(4 \mathrm{~mL})$, whirled up and left for deposition. The pentane phase was withdrawn, and this washing procedure was repeated twice with two further batches of pentane. The washed precipitate dissolved completely during the dropwise addition of a solution of dichloride $2 \mathbf{c}(470 \mathrm{mg}, 1.84 \mathrm{mmol})$ in THF $(5.0 \mathrm{~mL})$ with stirring at room temperature. After $2 \mathrm{~h}$, the contents of the Schlenk flask were 
poured onto solid $\mathrm{CO}_{2}$ and, after warming to room temperature, dissolved in $2 \mathrm{M} \mathrm{NaOH}(20 \mathrm{~mL})$ plus $\mathrm{Et}_{2} \mathrm{O}(40 \mathrm{~mL})$. The $\mathrm{NaOH}$ layer was extracted with more $\mathrm{Et}_{2} \mathrm{O}(20 \mathrm{~mL})$ and then acidified, delivering no organic acid. The combined $\mathrm{Et}_{2} \mathrm{O}$ layers were washed with pure water until neutral, dried over $\mathrm{Na}_{2} \mathrm{SO}_{4}$, and concentrated to give $810 \mathrm{mg}$ of a mixture (mp 129-134 ${ }^{\circ} \mathrm{C}$ ) of the crude product $4 \mathbf{p}(78 \%$ yield by NMR) along with chloro-2,4,6-tri-tert-butylbenzene (ClMes*, 8\%), HMes* (9\% with respect to $\mathbf{4 p}$ ), an unidentified tetramethylindan derivative, and a tiny amount of reagent $\mathbf{2 c}$. The mixture was extracted with boiling ethanol (22 mL), leaving $85 \mathrm{mg}(10 \%)$ of almost pure 4p with mp 173.5-175.5 ${ }^{\circ} \mathrm{C}$. The cooled ethanol extract deposited $506 \mathrm{mg}$ (59\%) of a second modification of $\mathbf{4 p}$ with mp 145$146.5{ }^{\circ} \mathrm{C}$. Analytically pure $4 \mathbf{r}$, recrystallized twice from hexane, melted first at $148-149.5{ }^{\circ} \mathrm{C}$ but had a second mp $177-178{ }^{\circ} \mathrm{C}$ after resolidification; the NMR spectra of both modifications were identical. $\quad{ }^{1} \mathrm{H}$ NMR (400 MHz, $\mathrm{CDCl}_{3}$, numbering of Scheme 8) $\delta 1.14\left(\mathrm{~s}, 2\right.$ 3- $\left.\mathrm{CH}_{3}\right), 1.33(\mathrm{~s}, p$ $\left.\mathrm{CMe}_{3}\right), 1.52\left(\mathrm{~s}, 2 \mathrm{o}-\mathrm{CMe}_{3}\right), 1.72\left(\mathrm{~s}, 21-\mathrm{CH}_{3}\right), 6.98\left(\mathrm{dm},{ }^{3} \mathrm{~J}=7 \mathrm{~Hz}, 4-\mathrm{H}\right), 7.16(\mathrm{~m}, 7-\mathrm{H}), 7.18(\mathrm{~m}, 5-\mathrm{H})$, $7.22\left(\mathrm{td},{ }^{3} J \approx 7 \mathrm{~Hz},{ }^{4} J \approx 1.4 \mathrm{~Hz}, 6-\mathrm{H}\right), 7.42(\mathrm{~s}, 2 m-\mathrm{H})$, assigned by the NOESY correlations $o-t-\mathrm{Bu} \leftrightarrow$ $m-\mathrm{H} \leftrightarrow p-t-\mathrm{Bu}, o-t-\mathrm{Bu} \leftrightarrow 1-\mathrm{CH}_{3} \leftrightarrow 7-\mathrm{H} \leftrightarrow 6-\mathrm{H}, o-t-\mathrm{Bu} \leftrightarrow 3-\mathrm{CH}_{3} \leftrightarrow 4-\mathrm{H} \leftrightarrow 5-\mathrm{H} ;{ }^{13} \mathrm{C}$ NMR (100.6 $\left.\mathrm{MHz} \mathrm{CDCl}_{3}\right) \delta 26.64\left(\mathrm{qq},{ }^{1} J=127.4 \mathrm{~Hz},{ }^{3} J=4.6 \mathrm{~Hz}, 21-\mathrm{CH}_{3}\right), 30.73\left(\mathrm{qq},{ }^{1} J=127.4 \mathrm{~Hz},{ }^{3} J=4.6\right.$ $\mathrm{Hz}, 23-\mathrm{CH}_{3}$ ), 31.32 (qsept, ${ }^{1} J=125.5 \mathrm{~Hz},{ }^{3} J=4.9 \mathrm{~Hz}, p-\mathrm{CMe} e_{3}$ ), 34.24 (qsept, ${ }^{1} J=125.8 \mathrm{~Hz},{ }^{3} J=4.8$ $\mathrm{Hz}, 2 o-\mathrm{CMe}_{3}$ ), 34.64 (partially hidden m, p-CMe $), 39.60$ (m, $\left.2 o-C \mathrm{Me}_{3}\right), 49.40\left(\mathrm{~m}, \mathrm{C}^{3}\right), 50.52(\mathrm{~m}$, $\left.\mathrm{C}^{1}\right), 121.88\left(\mathrm{dm},{ }^{1} J=156 \mathrm{~Hz}, \mathrm{C}^{4}\right), 122.41\left(\mathrm{dm},{ }^{1} J=156 \mathrm{~Hz}, \mathrm{C}^{7}\right), 124.06\left(\mathrm{dd},{ }^{1} J=152.3 \mathrm{~Hz},{ }^{3} J=6.8\right.$ $\left.\mathrm{Hz}, 2 \mathrm{C}^{m}\right), 126.99\left(\mathrm{dm},{ }^{1} J=159 \mathrm{~Hz}, \mathrm{C}^{5}\right), 127.04\left(\mathrm{dm},{ }^{1} J=159 \mathrm{~Hz}, \mathrm{C}^{6}\right), 129.00\left(\operatorname{sharp~s}, \mathrm{C}^{\alpha}\right), 130.74(\mathrm{t}$, $\left.{ }^{3} J=7.2 \mathrm{~Hz}, \mathrm{C}^{i}\right), 148.15\left(\mathrm{~m},{ }^{3} J=3.3 \mathrm{~Hz}, 2 \mathrm{C}^{o}\right), 149.00\left(\mathrm{~m}, \mathrm{C}^{2}\right), 149.24\left(\mathrm{~m}, \mathrm{C}^{9}\right), 149.58\left(\mathrm{~m},{ }^{3} J \approx 3.7 \mathrm{~Hz}\right.$ $\left.\mathrm{C}^{p}\right), 149.61\left(\mathrm{~m}, \mathrm{C}^{8}\right) ; \mathrm{CH}$ and $\mathrm{CH}_{3}$ resonances were assigned by ${ }^{1} \mathrm{H} /{ }^{13} \mathrm{C}$ heterocorrelation and confirmed by COLOCS $\left({ }^{13} \mathrm{C},{ }^{1} \mathrm{H}\right.$ with window $\left.6 \mathrm{~Hz}\right)$ which identified also the signals of carbon atoms carrying no hydrogen by the ${ }^{2} J_{\mathrm{CH}}$ correlations $\mathrm{C}^{1} \leftrightarrow 1-\mathrm{CH}_{3}, \mathrm{C}^{3} \leftrightarrow 3-\mathrm{CH}_{3}, o-C \mathrm{Me}_{3} \leftrightarrow o-\mathrm{CMe}_{3}$, and $p-C \mathrm{Me}_{3} \leftrightarrow p-$ $\mathrm{CMe}_{3}$, in addition to the ${ }^{3} J_{\mathrm{CH}}$ correlations $\mathrm{C}^{5} \leftrightarrow 7-\mathrm{H} \leftrightarrow \mathrm{C}^{9} \leftrightarrow 3-\mathrm{CH}_{3} \leftrightarrow \mathrm{C}^{2} \leftrightarrow 1-\mathrm{CH}_{3} \leftrightarrow \mathrm{C}^{8} \leftrightarrow 4-\mathrm{H}$ $\leftrightarrow \mathrm{C}^{6}, \mathrm{C}^{o} \leftrightarrow o-\mathrm{CMe}_{3}, \mathrm{C}^{p} \leftrightarrow p-\mathrm{CMe}_{3}, \mathrm{C}^{i} \leftrightarrow m-\mathrm{H} \leftrightarrow \mathrm{C}^{m}, \mathrm{C}^{4} \leftrightarrow 6-\mathrm{H}, \mathrm{C}^{7} \leftrightarrow 5-\mathrm{H}$, and $o-C \mathrm{Me}_{3} \leftrightarrow m-\mathrm{H}$ $\leftrightarrow p-\mathrm{Me}_{3}$; IR (KBr) 2966 (s), 2869, 1486, 1366, and $754 \mathrm{~cm}^{-1}$. Anal. Calcd for $\mathrm{C}_{32} \mathrm{H}_{45} \mathrm{Cl}$ (465.16): $\mathrm{C}, 82.63 ; \mathrm{H}, 9.75 ; \mathrm{Cl}, 7.62$. Found: $\mathrm{C}, 83.06 ; \mathrm{H}, 9.79 ; \mathrm{Cl}, 7.52$. - This substitution reaction is much slower in $\mathrm{Et}_{2} \mathrm{O}$ at room temperature $\left(t_{1 / 2} \approx 0.5 \mathrm{~h}\right) .{ }^{16}$

2-(1,1,3,3-Tetramethyl-2-indanylidene)hexanoic Acid (10i). The combined acid fractions from several experiments were distilled at 0.025 mbar to afford a forerun of $10 a\left(90-100{ }^{\circ} \mathrm{C}\right.$ bath temperature) and then the main component $10 \mathrm{i}\left(132-140{ }^{\circ} \mathrm{C}\right.$ bath) which was recrystallized from $\mathrm{CCl}_{4}$ : Colorless platelets, mp 168-169 ${ }^{\circ} \mathrm{C} ;{ }^{1} \mathrm{H}$ NMR (400 MHz, $\mathrm{CDCl}_{3}$, numbering of Scheme 6) $\delta 0.96(\mathrm{t}$, $\left.{ }^{3} J=7.1 \mathrm{~Hz}, 3 \varepsilon-\mathrm{H}\right), 1.44$ and $1.50(2 \mathrm{~m}, 2+2 \delta-/ \gamma-\mathrm{H}), 1.54$ and $1.56\left(2 \mathrm{~s}, 2+21-/ 3-\mathrm{CH}_{3}\right), 2.62$ 
(pseudo-t, $\left.{ }^{3} J \approx 7.9 \mathrm{~Hz}, 2 \beta-\mathrm{H}\right), 7.13$ and 7.15 (2 m, 4-/7-H), 7.24 (m, 5-/6-H); ${ }^{13} \mathrm{C}$ NMR (100.6 MHz, $\left.\mathrm{CDCl}_{3}\right) \delta 14.04\left(\mathrm{C}^{\varepsilon}\right), 22.75\left(\mathrm{C}^{\delta}\right), 30.15$ and $30.44\left(2+21-/ 3-\mathrm{CH}_{3}\right), 30.64\left(\mathrm{C}^{\gamma}\right), 31.40\left(\mathrm{C}^{\beta}\right), 47.69$ and $48.26\left(\mathrm{C}^{1,3}\right), 122.15$ and $122.18\left(\mathrm{C}^{4,7}\right), 127.11$ and $127.23\left(\mathrm{C}^{5,6}\right), 128.75\left(\mathrm{C}^{\alpha}\right), 149.20$ and $149.69\left(\mathrm{C}^{8,9}\right)$, $158.12\left(\mathrm{C}^{2}\right), 175.75\left(\mathrm{CO}_{2} \mathrm{H}\right)$; IR (KBr) 3600-2500 (br, O-H), 2962, 2928, 2870, $1690(\mathrm{~s}, \mathrm{C}=\mathrm{O})$, 1283, and $756 \mathrm{~cm}^{-1}$. Anal. Calcd for $\mathrm{C}_{19} \mathrm{H}_{26} \mathrm{O}_{2}$ (286.4): C, 79.68; H, 9.15. Found: C, 79.39; H, 9.23 .

\section{Scheme 6}

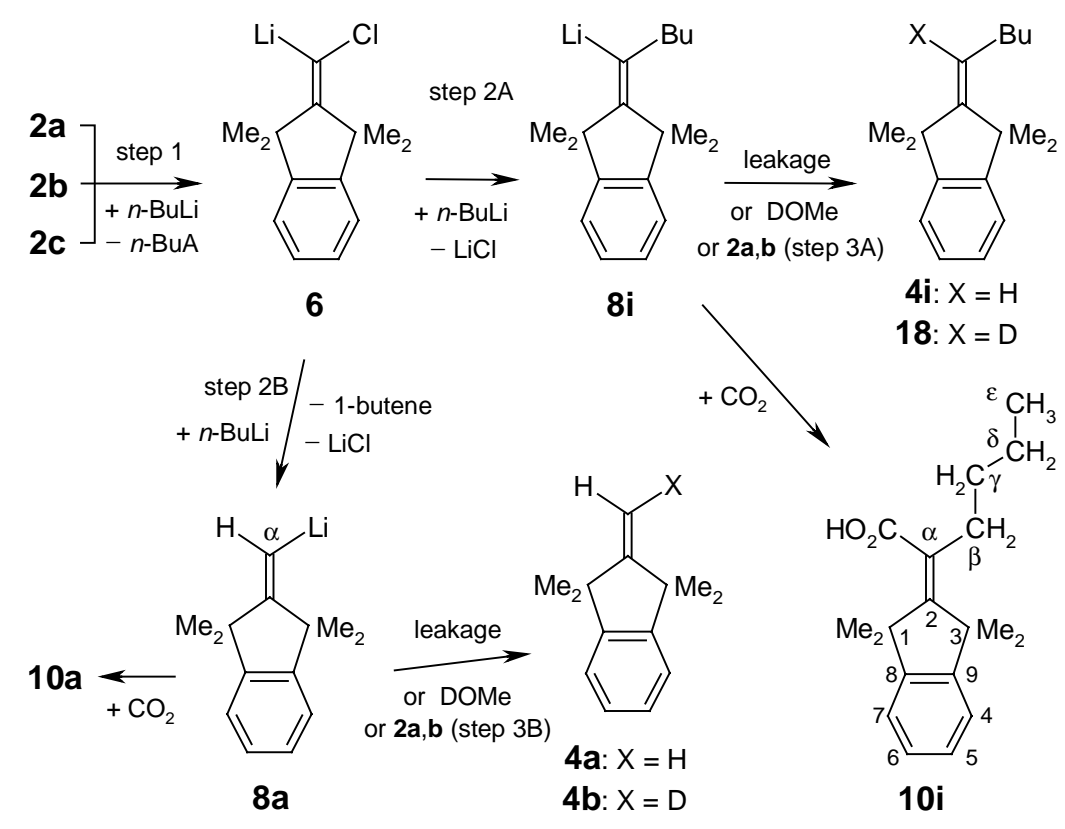

2-(1-Methyl-3-phenyl-2-propyn-1-ylidene)-1,1,3,3-tetramethylindan (16). Phenylethynyllithium ( $0.20 \mathrm{mmol}, 1.0$ equiv), prepared in a dry NMR tube $(5 \mathrm{~mm})$ at $-78{ }^{\circ} \mathrm{C}$ under argon cover gas from phenylacetylene $(0.022 \mathrm{~mL}, 0.20 \mathrm{mmol})$ in THF $(0.5 \mathrm{~mL})$ with $n$-BuLi (1 equiv) in hexane $(0.10 \mathrm{~mL})$, did not react with dichloride $2 \mathrm{c}(50 \mathrm{mg}, 0.196 \mathrm{mmol})$ at ambient temperature over three days. After addition at $-78{ }^{\circ} \mathrm{C}$ of one portion of $\mathrm{MeLi}\left(0.22 \mathrm{mmol}, 1.1\right.$ equiv) in $\mathrm{Et}_{2} \mathrm{O}(0.17 \mathrm{~mL})$, the ${ }^{1} \mathrm{H}$ NMR singlet of MeLi was observed for $125 \mathrm{~min}$ at room temperature and had dropped to zero overnight. The solution was poured onto solid $\mathrm{CO}_{2}$, warmed up, and dissolved in $\mathrm{Et}_{2} \mathrm{O}$ plus $2 \mathrm{M} \mathrm{NaOH}$. The acidified $\mathrm{NaOH}$ phase afforded $\mathrm{PhC} \equiv \mathrm{CCO}_{2} \mathrm{H}(75 \%)$. The $\mathrm{Et}_{2} \mathrm{O}$ phase was washed until neutral, dried over $\mathrm{MgSO}_{4}$, and concentrated to yield a solidifying 2:1 mixture (38 mg) of 16 and $\mathbf{4 f}$. Pale yellow, pure 16 was obtained through recrystallizations from methanol: mp $115-116{ }^{\circ} \mathrm{C}$; ${ }^{1} \mathrm{H} \mathrm{NMR} \mathrm{(400} \mathrm{MHz}, \mathrm{CDCl}_{3}$, numbering of Scheme 6) $\delta 1.55\left(\mathrm{~s}, 23-\mathrm{CH}_{3}\right), 1.71\left(\mathrm{~s}, 2 \mathrm{-}-\mathrm{CH}_{3}\right), 2.20\left(\mathrm{~s}, \alpha-\mathrm{CH}_{3}\right), 7.17(\mathrm{~m}, 4-/ 7-\mathrm{H})$, $7.24(\mathrm{~m}, 5-/ 6-\mathrm{H}), 7.33(\mathrm{~m}, 2 \mathrm{~m}-\mathrm{H}$ and $1 \mathrm{p}-\mathrm{H})$, and $7.47\left(\mathrm{dm},{ }^{3} \mathrm{~J}=8 \mathrm{~Hz}, 2 o-\mathrm{H}\right) ;{ }^{13} \mathrm{C} \mathrm{NMR}(100.6 \mathrm{MHz}$, $\left.\mathrm{CDCl}_{3}\right) \delta 21.19\left(\alpha-\mathrm{CH}_{3}\right), 28.86\left(21-\mathrm{CH}_{3}\right), 29.13\left(23-\mathrm{CH}_{3}\right), 48.16\left(\mathrm{C}^{3}\right), 48.62\left(\mathrm{C}^{1}\right), 92.27\left(\mathrm{C}^{\beta}\right), 93.80$ $\left(\mathrm{C}^{\gamma}\right), 111.76\left(\mathrm{C}^{\alpha}\right), 122.23$ and $122.47\left(\mathrm{C}^{4,7}\right), 124.21\left(\mathrm{C}^{\mathrm{i}}\right), 127.02$ and $127.11\left(\mathrm{C}^{5,6}\right), 127.78\left(\mathrm{C}^{p}\right), 128.35$ 
$\left(\mathrm{C}^{m}\right), 130.86\left(\mathrm{C}^{o}\right), 149.82\left(\mathrm{C}^{9}\right), 149.93\left(\mathrm{C}^{8}\right), 165.15\left(\mathrm{C}^{2}\right)$, assigned by comparison with $4 \mathrm{~g} ; \mathrm{IR}(\mathrm{KBr})$ 2962, 1486, 1021, 770, 756 (s), and $689 \mathrm{~cm}^{-1}$. Anal. Calcd for $\mathrm{C}_{23} \mathrm{H}_{24}$ (300.4): C, 91.95; H, 8.05. Found: C, 91.35; H, 8.08.

2-(1,3-Diphenyl-2-propyn-1-ylidene)-1,1,3,3-tetramethylindan (20). The constitution was verified by treatment of the chloroalkene $\mathbf{4 h}$ at room temperature in THF with $\mathrm{PhLi}$. The in situ ${ }^{1} \mathrm{H}$ NMR spectra revealed that no product was formed within $50 \mathrm{~min}$ but that $\mathrm{PhLi}$ vanished overnight. The nonacidic portion isolated after carboxylation contained 20 with the characteristic ${ }^{1} \mathrm{H}$ NMR resonances: $\delta$ (400 MHz, $\left.\mathrm{CDCl}_{3}\right) 1.54$ and $1.94\left(2 \mathrm{~s}, 2+2\right.$ 1-/3- $\left.\mathrm{CH}_{3}\right), 7.58$ (m, 4 aromat. $\left.\mathrm{H}\right)$.

\section{Side-products S7 - S10 Formed from 2-(1-Chloroethylidene)-1,1,3,3-tetramethylindan (4f).}

\section{Scheme S2}
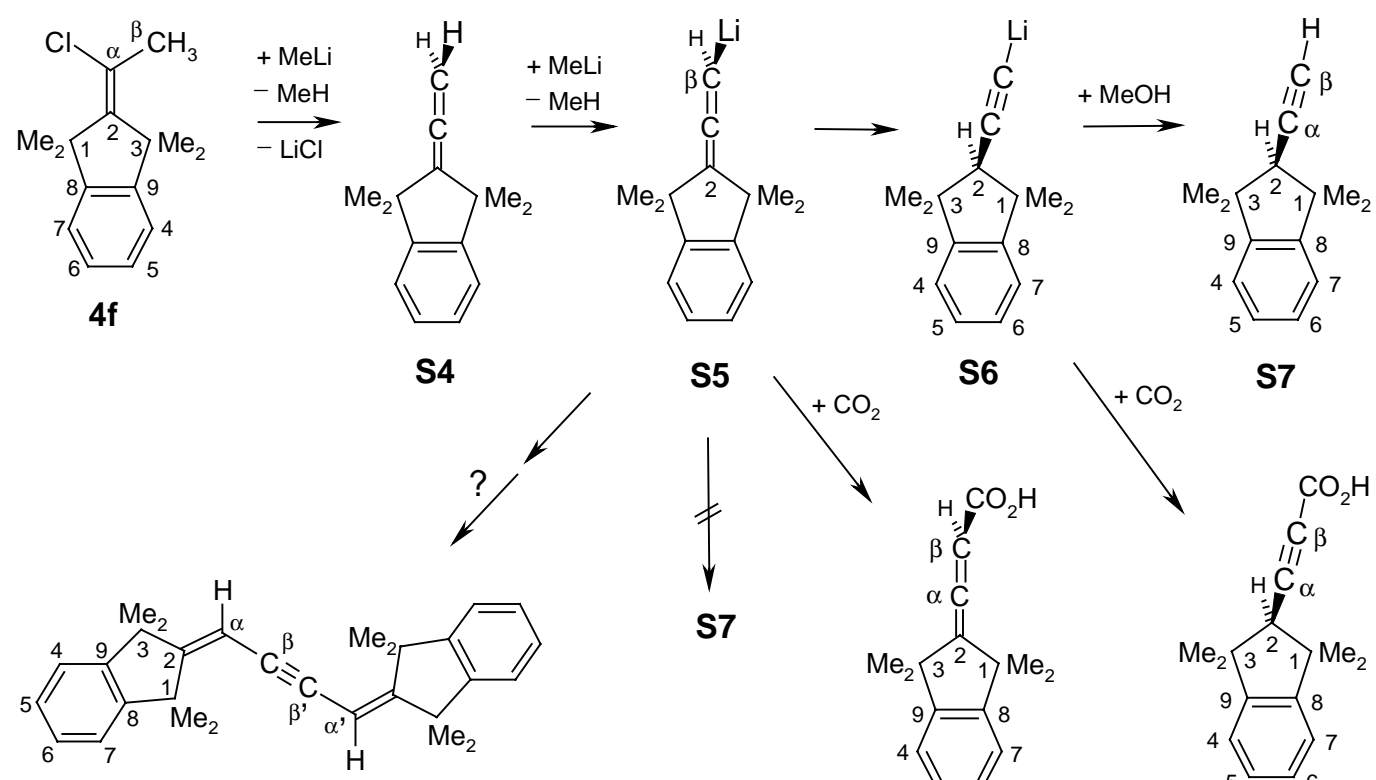

S8

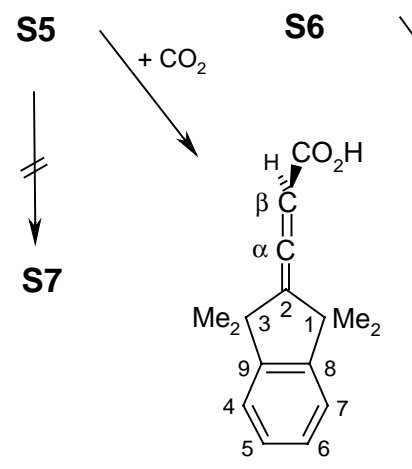

S9

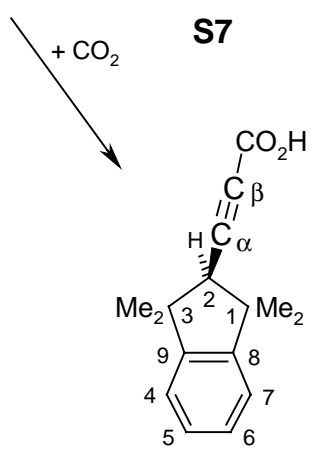

S10

The product $\mathbf{4 f}$ of chlorine transfer from $\mathbf{2 c}$ to $\mathbf{8 d}$ can undergo the following slow changes in the presence of an excess of MeLi in THF. The elimination of $\mathrm{HCl}$ to produce an allene (S4) is not unusual $^{\mathrm{S} 16}$ and was followed here by the faster deprotonation of S4 to give S5, as shown by carboxylation which furnished the allenecarboxylic acid S9 (10\% yield) already after one night, accompanied only by residual $\mathbf{4 f}$ but no allene $\mathbf{S 4}$. The deprotonation of other allenes was reported to occur rapidly with $n$-butyllithium in $\mathrm{THF}$ at $-50{ }^{\circ} \mathrm{C},{ }^{\mathrm{S} 17}$ but slowly with $\mathrm{MeLi}$ in $\mathrm{Et}_{2} \mathrm{O}^{\mathrm{S} 18}$ at room temperature. The ensuing formal $\beta$,2-hydrogen shift in $\mathbf{S 5}$ to give $\mathbf{S 6}$ has precedence in slowly ${ }^{\mathrm{S} 18-\mathrm{S} 20}$ or 
rapidly $^{\mathrm{S} 21}$ occurring similar isomerizations and is probably ${ }^{\mathrm{S} 19, \mathrm{~S} 21}$ catalyzed by the 2, $\beta$-dilithio intermediate. Bimolecular mechanisms such as these would predict the formation of $\mathbf{S 6}$ to be retarded by steric shielding, in keeping with the isolation of S7 (quantitative) or of S10 (69\%) after at least one week at room temperature.

2-Ethynyl-1,1,3,3-tetramethylindan (S7). MeLi (6.27 mmol) in $\mathrm{Et}_{2} \mathrm{O}(5.06 \mathrm{~mL})$ was added at -78 ${ }^{\circ} \mathrm{C}$ under argon cover gas to the dichloride $2 \mathrm{c}$ (400 mg, $\left.1.57 \mathrm{mmol}\right)$ in anhydrous THF (5.0 mL). After one week at room temperature, the black solution was cooled, protonated with methanol, and diluted with water and $\mathrm{Et}_{2} \mathrm{O}(75 \mathrm{~mL})$. The washed and dried $\left(\mathrm{MgSO}_{4}\right) \mathrm{Et}_{2} \mathrm{O}$ layer was concentrated to yield the almost pure alkyne S7 (310 mg, 100\%). Its solution in ethanol precipitated a colorless powder (20 mg) with the structure S8 of a rearranged "dehydro-dimer" of S5 or S6. The remaining material was distilled at $120-140{ }^{\circ} \mathrm{C}$ (bath temp.)/12 Torr to afford S7 as a colorless oil (131 mg, 28\%), which crystallized from methanol at $-78{ }^{\circ} \mathrm{C}: \operatorname{mp} 35-36{ }^{\circ} \mathrm{C} ;{ }^{1} \mathrm{H}$ NMR $\left(400 \mathrm{MHz}, \mathrm{CDCl}_{3}\right) \delta 1.29$ and $1.39(2$ s, $\left.2+21-/ 3-\mathrm{CH}_{3}\right), 2.29\left(\mathrm{~d},{ }^{4} \mathrm{~J}=2.7 \mathrm{~Hz}, 2-\mathrm{H}\right), 2.79\left(\mathrm{~d},{ }^{4} \mathrm{~J}=2.7 \mathrm{~Hz}, \equiv \mathrm{C}-\mathrm{H}\right), 7.15$ and $7.22\left(2 \mathrm{~m}, \mathrm{C}_{6} \mathrm{H}_{4}\right)$; ${ }^{13} \mathrm{C} \mathrm{NMR}\left(100.6 \mathrm{MHz}, \mathrm{CDCl}_{3}\right) \quad \delta 27.51$ (qqi, ${ }^{1} \mathrm{~J}=126.8 \mathrm{~Hz},{ }^{3} \mathrm{~J}=4.7 \mathrm{~Hz}, 1-/ 3-\mathrm{CH}_{3}$ trans or cis to $\mathrm{C} \equiv \mathrm{C}$ ), 29.35 (qqi, ${ }^{1} J=126.0 \mathrm{~Hz},{ }^{3} J=4.9 \mathrm{~Hz}, 1-/ 3-\mathrm{CH}_{3}$ cis or trans to $\mathrm{C} \equiv \mathrm{C}$ ), 45.40 (unresolved, $\mathrm{C}^{1,3}$ ), $54.79\left(\mathrm{dm},{ }^{1} J=129.2 \mathrm{~Hz}, \mathrm{C}^{2}\right), 73.34\left(\mathrm{dd},{ }^{1} J=246.8 \mathrm{~Hz},{ }^{3} J=4.1 \mathrm{~Hz}, \mathrm{C}^{\beta}\right), 82.38\left(\mathrm{dd},{ }^{2} J=49.0 \mathrm{~Hz},{ }^{2} J=\right.$ $\left.10.5 \mathrm{~Hz}, \mathrm{C}^{\alpha}\right), 122.71\left(\mathrm{dm},{ }^{1} J=156 \mathrm{~Hz}, \mathrm{C}^{4,7}\right), 127.05\left(\mathrm{dd},{ }^{1} J=159 \mathrm{~Hz},{ }^{3} J=7.2 \mathrm{~Hz}, \mathrm{C}^{5,6}\right), 149.25\left(\mathrm{~m},{ }^{3} J\right.$ $\left.\approx 3.7 \mathrm{~Hz}, \mathrm{C}^{8,9}\right) ; \quad \mathrm{IR}(\mathrm{KBr}) 3308(\equiv \mathrm{C}-\mathrm{H}), 2962,2866,2113(\mathrm{w}, \mathrm{C} \equiv \mathrm{C}), 1481$, and $756(\mathrm{~s}) \mathrm{cm}^{-1}$. Anal. Calcd for $\mathrm{C}_{15} \mathrm{H}_{18}$ (198.3): C, 90.85; H, 9.15. Found: C, 91.01; H, 9.11.

1,4-Bis(1,1,3,3-tetramethyl-2-indanylidene)-2-butyne (S8). This high-melting “dehydro-dimer" (> $240{ }^{\circ} \mathrm{C}$ ) was isolated in a very low yield $(20 \mathrm{mg})$ from the crude material containing the alkyne S7. It may have been formed by an oxydative dimerization of the alkenyllithium (S5) or the alkynyllithium (S6) intermediate and subsequent tautomerizations. It reminds of earlier observations by Ludvig and Lagow who reported ${ }^{\mathrm{S} 22}$ that $\mathrm{Ph}_{2} \mathrm{C}=\mathrm{CHLi}$ furnished $\mathrm{Ph}_{2} \mathrm{C}=\mathrm{CH}-\mathrm{CH}=\mathrm{CPh}_{2}$ as the main product through pyrolysis at $+125{ }^{\circ} \mathrm{C}$ (or "in smaller amounts when kept in THF solution"). ${ }^{1} \mathrm{H}$ NMR (400 MHz, $\left.\mathrm{CDCl}_{3}\right) \delta 1.41\left(\mathrm{~s}, 3-\mathrm{CH}_{3}\right), 1.66\left(\mathrm{~s}, 1-\mathrm{CH}_{3}\right), 5.86(\mathrm{~s}, \alpha-\mathrm{H}), 7.18(\mathrm{~m}, 4-\mathrm{H}), 7.21(\mathrm{~m}, 7-\mathrm{H}), 7.25(\mathrm{~m}, 5-/ 6-$ $\mathrm{H})$, assigned by the NOESY correlations $\alpha-\mathrm{H} \leftrightarrow 3-\mathrm{CH}_{3} \leftrightarrow 4-\mathrm{H}$ and $1-\mathrm{CH}_{3} \leftrightarrow 7-\mathrm{H} ;{ }^{13} \mathrm{C}$ NMR (100.6 $\left.\mathrm{MHz} \mathrm{CDCl}_{3}\right) \delta 28.7\left(\mathrm{qq},{ }^{1} J=127.4 \mathrm{~Hz},{ }^{3} J=4.6 \mathrm{~Hz}, 1-\mathrm{CH}_{3}\right), 32.0\left(\mathrm{qq},{ }^{1} J=127.4 \mathrm{~Hz},{ }^{3} J=4.6 \mathrm{~Hz}, 3-\right.$ $\left.\mathrm{CH}_{3}\right), 47.85\left(\mathrm{ddm},{ }^{3} J=8.5 \mathrm{~Hz},{ }^{3} J=1.5 \mathrm{~Hz}, \mathrm{C}^{1}\right), 47.97\left(\mathrm{~m}, \mathrm{C}^{3}\right), 93.8\left(\mathrm{X}\right.$ part of AA $\mathrm{X}$ with ${ }^{2} J_{\mathrm{AX}}=-1.07$ $\mathrm{Hz},{ }^{3} J_{\mathrm{A}^{\prime} \mathrm{X}}=4.78 \mathrm{~Hz}$, and ${ }^{5} J_{\mathrm{AA}^{\prime}}=+2.81 \mathrm{~Hz}, \mathrm{C}^{\beta}$ coupled to both $\mathrm{A}=\alpha-\mathrm{H}$ and $\mathrm{A}^{\prime}=\alpha^{\prime}-\mathrm{H}$ as analysed through spectrum simulation), $101.7\left(\mathrm{dd},{ }^{1} J=158.9 \mathrm{~Hz},{ }^{4} J=1.9 \mathrm{~Hz}, \mathrm{C}^{\alpha}\right), 122.51$ and $122.52(2 \mathrm{dm}$, both with $\left.{ }^{1} J=155.6 \mathrm{~Hz}, \mathrm{C}^{4,7}\right), 127.1$ and $127.3\left(2 \mathrm{ddd}\right.$, both with $\left.{ }^{1} J=158.8 \mathrm{~Hz},{ }^{3} J=7 \mathrm{~Hz}, \mathrm{C}^{5,6}\right), 148.3$ $\left(\mathrm{tm},{ }^{3} J=6.1 \mathrm{~Hz}, \mathrm{C}^{9}\right), 149.9\left(\mathrm{tm},{ }^{3} J=6.2 \mathrm{~Hz}, \mathrm{C}^{8}\right), 172.8\left(\mathrm{~m}, \mathrm{C}^{2}\right)$; IR (KBr) 2960, 2929, 2860, 1480, 
1450, 1110, and $760 \mathrm{~cm}^{-1}$. The constitution of $\mathbf{S 8}$ followed from its spectral $C_{2 \mathrm{~h}}$ symmetry, combined with the unusual $\mathrm{CH}$ coupling pattern of ${ }^{13} \mathrm{C}^{\beta}$ as caused by two isochronous protons $\left(\alpha-\mathrm{H}\right.$ and $\left.\alpha^{\prime}-\mathrm{H}\right)$ which was confirmed through spectrum simulation and selective decoupling of $\alpha-\mathrm{H}\left(\rightarrow \mathrm{C}^{\beta}\right.$ became a singlet).

3-(1,1,3,3-Tetramethyl-2-indanylidene)propenoic Acid (S9). $\mathrm{MeLi}(3.13 \mathrm{mmol})$ in $\mathrm{Et}_{2} \mathrm{O}(2.53$ $\mathrm{mL}$ ) was added at $-78{ }^{\circ} \mathrm{C}$ under argon cover gas to a stirred solution of dichloride $2 \mathrm{c}$ (400 mg, 1.57 $\mathrm{mmol})$ in anhydrous THF $(4.0 \mathrm{~mL})$. After one night at room temperature, the solution was poured onto solid $\mathrm{CO}_{2}$, warmed up, and diluted with $\mathrm{Et}_{2} \mathrm{O}$ plus $2 \mathrm{M} \mathrm{NaOH}$. The $\mathrm{Et}_{2} \mathrm{O}$ phase was washed until neutral, dried over $\mathrm{MgSO}_{4}$, filtered, and concentrated to afford the almost pure chloroalkene $4 \mathbf{f}$ (280 $\mathrm{mg}, 76 \%$ ). The acidified $\mathrm{NaOH}$ layer was extracted with $\mathrm{Et}_{2} \mathrm{O}$ to give the hardly contaminated allenecarboxylic acid S9 (38 mg, 10\%): Colorless powder, mp 204-205 ${ }^{\circ} \mathrm{C}\left(\mathrm{CCl}_{4}\right)$; ${ }^{1} \mathrm{H}$ NMR (400 $\left.\mathrm{MHz}_{,} \mathrm{CDCl}_{3}\right) \delta 1.45$ and $1.49\left(2 \mathrm{~s}, 2+21-/ 3-\mathrm{CH}_{3}\right), 5.82(\mathrm{~s}, \beta-\mathrm{H}), 7.16$ and $7.26\left(2 \mathrm{~m}, \mathrm{C}_{6} \mathrm{H}_{4}\right) ;{ }^{13} \mathrm{C}$ NMR $\left(100.6 \mathrm{MHz}, \mathrm{CDCl}_{3}\right) \quad \delta 30.86$ and $31.01\left(2+21-/ 3-\mathrm{CH}_{3}\right), 48.21\left(\mathrm{C}^{1,3}\right), 92.61\left(\mathrm{C}^{\beta}\right), 122.45$ $\left(\mathrm{C}^{4,7}\right), 127.58\left(\mathrm{C}^{5,6}\right), 128.70\left(\mathrm{C}^{2}\right), 148.04\left(\mathrm{C}^{8,9}\right), 171.43\left(\mathrm{CO}_{2} \mathrm{H}\right), 208.44\left(\mathrm{C}^{\alpha}\right) ; \mathrm{IR}(\mathrm{KBr})$ 3600-2400 (O-H), 2965, 1958 (s, C=C=C), 1666 (vs, C=O), 1294, and 761 (s) cm ${ }^{-1}$. Anal. Calcd for $\mathrm{C}_{16} \mathrm{H}_{18} \mathrm{O}_{2}$ (242.3): C, 79.31; H, 7.49. Found: C, 79.23; H, 7.45.

3-(1,1,3,3-Tetramethyl-2-indanyl)propiolic Acid (S10). MeLi (6.20 mmol) in $\mathrm{Et}_{2} \mathrm{O}(5.0 \mathrm{~mL}) \mathrm{was}$ added at $-78{ }^{\circ} \mathrm{C}$ under argon cover gas to a stirred solution of dichloride $2 \mathrm{c}$ (100 $\left.\mathrm{mg}, 0.39 \mathrm{mmol}\right)$ in anhydrous THF $(5.0 \mathrm{~mL})$. After two weeks at room temperature, the solution was poured onto solid $\mathrm{CO}_{2}$, warmed up, and diluted with $\mathrm{Et}_{2} \mathrm{O}$ plus $2 \mathrm{M} \mathrm{NaOH}$. The washed and dried $\mathrm{Et}_{2} \mathrm{O}$ layer furnished a mixture $\left(<20 \mathrm{mg}\right.$ ) of the ethylidene compound $\mathbf{4 d}$ and very little alkyne $\mathbf{S 7}$. The $\mathrm{Et}_{2} \mathrm{O}$ extract of the acidified $\mathrm{NaOH}$ phase was washed until neutral, dried over $\mathrm{MgSO}_{4}$, concentrated, and crystallized from low-boiling petroleum ether: $44 \mathrm{mg}(47 \%)$, mp 176-177 ${ }^{\circ} \mathrm{C} ;{ }^{1} \mathrm{H} \mathrm{NMR}\left(400 \mathrm{MHz}, \mathrm{CDCl}_{3}\right) \delta 1.34$ and $1.43\left(2 \mathrm{~s}, 2+2\right.$ 1-/3- $\left.\mathrm{CH}_{3}\right), 2.95(\mathrm{~s}, 2-\mathrm{H}), 7.15$ and $7.24\left(2 \mathrm{~m}, \mathrm{C}_{6} \mathrm{H}_{4}\right) ;{ }^{13} \mathrm{C} \mathrm{NMR}\left(100.6 \mathrm{MHz}, \mathrm{CDCl}_{3}\right) \delta$ 27.79 and $29.57\left(2+21-/ 3-\mathrm{CH}_{3}\right), 46.33\left(\mathrm{C}^{1,3}\right), 54.78\left(\mathrm{C}^{2}\right), 77.66\left(\mathrm{C}^{\beta}\right), 90.71\left(\mathrm{C}^{\alpha}\right), 122.66\left(\mathrm{C}^{4,7}\right)$, $127.39\left(\mathrm{C}^{5,6}\right), 148.55\left(\mathrm{C}^{8,9}\right), 157.22\left(\mathrm{CO}_{2} \mathrm{H}\right)$; IR (KBr) 3500-2500 (O-H), 2964, $2231(\mathrm{~s}, \mathrm{C} \equiv \mathrm{C}), 1682$ (vs, $\mathrm{C}=\mathrm{O}$ ), 1410, 1279, 753 (s) $\mathrm{cm}^{-1}$. Anal. Calcd for $\mathrm{C}_{16} \mathrm{H}_{18} \mathrm{O}_{2}$ (242.3): C, 79.31; H, 7.49. Found: C, 78.95; H, 7.65. 


\section{Substitutions with $t$-BuC $\equiv$ CLi: $S 11-S 15$.}

\section{Scheme S3}

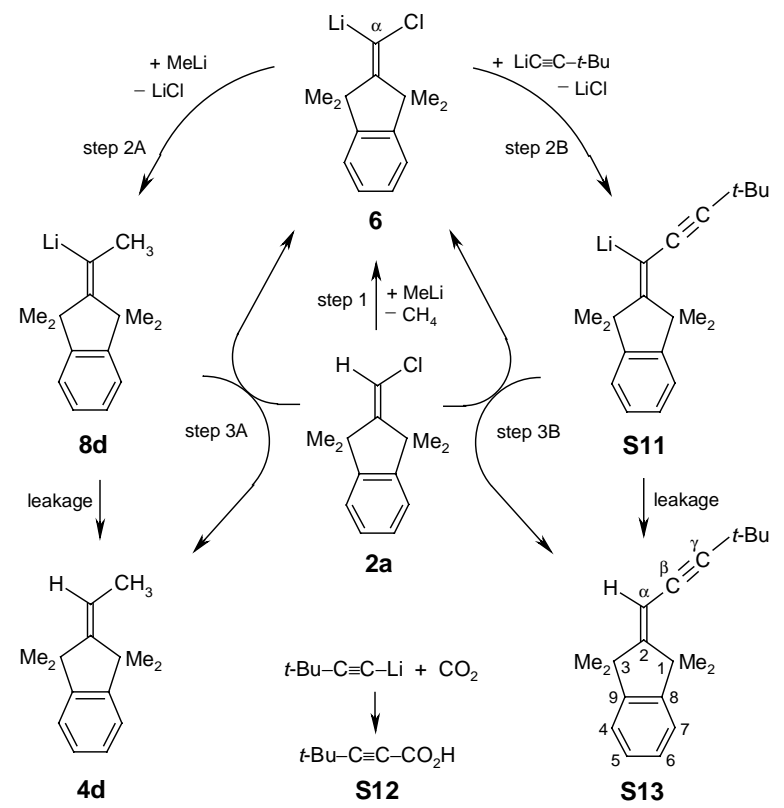

Methyllithium (MeLi) can react via the carbenoid chain route with both the $\alpha$-chloroalkene $2 \mathbf{a}$ and the $\alpha, \alpha$-dichloroalkene 2c, as established in the Main Text and depicted here in the left-hand sides of Schemes $\mathrm{S} 3$ and $\mathrm{S} 4$, respectively. $t$ - $\mathrm{BuC} \equiv \mathrm{CLi}$ alone is not sufficiently basic to react with reagents $2 \mathbf{a}$ or 2c, but it can compete (step 2B of Scheme S3) successfully with MeLi (step 2A) for substitution at $\mathrm{C}^{\alpha}$ of the carbenoid 6 that was generated by MeLi in the initiating step 1. The experiments were performed in analogy with those employing $\mathrm{PhC} \equiv \mathrm{CLi}$ and gave analogous results; but $t$-BuC $\equiv \mathrm{CLi}$ reacted slower than $\mathrm{PhC} \equiv \mathrm{CLi}$ by a factor of $2.1( \pm 0.2)$ according to competition experiments.

\subsection{Using Reagent $2 a$.}

The intermediate substitution products $\mathbf{S 1 1}$ and $\mathbf{8 d}$ are both suffiently basic to deprotonate reagent $\mathbf{2 a}$, so that carbenoid $\mathbf{6}$ is regenerated in the chain propagating steps 3B and 3A. The product $\mathbf{S 1 3}$ is formed from the chain carrier S11 not only via step 3B but also through protonation by adventitious sources other than 2a ("leakage" to S13); this interrupts the chain and halts the conversion until another batch of the initiator MeLi restarts the chain. Final carboxylation was intended to furnish $t$ $\mathrm{BuC} \equiv \mathrm{CCO}_{2} \mathrm{H}(\mathbf{S 1 2})$, in order to provide evidence that a sufficient amount of $t$-BuC $\equiv \mathrm{CLi}$ had remained in the solution.

2-(4,4-Dimethyl-2-pentyn-1-ylidene)-1,1,3,3-tetramethylindan (S13). Reagent 2a (110 mg, 0.50 mmol, $0.23 \mathrm{M})$ in $\mathrm{Et}_{2} \mathrm{O}$ plus $\mathrm{THF}(74: 26$ by volume) did not react with $t$ - $\mathrm{BuC} \equiv \mathrm{CLi}(0.46 \mathrm{M})$ at room 
temperature during one night. After the addition of MeLi (finally $0.30 \mathrm{M}$ ), $2 \mathbf{a}$ vanished in the course of $6 \mathrm{~h}$. After one further night, the mixture was poured onto solid $\mathrm{CO}_{2}$, warmed up to ambient temperature, and dissolved in $\mathrm{Et}_{2} \mathrm{O}$ plus $2 \mathrm{M} \mathrm{NaOH}$. The acidified $\mathrm{NaOH}$ phase furnished $42 \mathrm{mg}(0.33$ mmol) of pure $t$ - $\mathrm{BuC} \equiv \mathrm{CCO}_{2} \mathrm{H}(\mathbf{S 1 2})$. The non-acidic fraction $(67 \mathrm{mg}$ ) consisted of $\mathbf{S 1 3}$ (36\% yield) and 4d (19\%). The colorless oil S13 could not be purified completely through distillation at 120-130 ${ }^{\circ} \mathrm{C}$ (bath temperature)/1 mbar and subsequent chromatography on $\mathrm{SiO}_{2}$ with petroleum ether plus $\mathrm{Et}_{2} \mathrm{O}$ (10:1). ${ }^{1} \mathrm{H} \mathrm{NMR}\left(400 \mathrm{MHz}, \mathrm{CDCl}_{3}\right) \delta 1.31\left(\mathrm{~s}, \mathrm{CMe}\right.$ ) $, 1.35\left(\mathrm{~s}, 23-\mathrm{CH}_{3}\right), 1.62\left(\mathrm{~s}, 2\right.$ 1- $\left.\mathrm{CH}_{3}\right), 5.59(\mathrm{~s}, \alpha-$ $\mathrm{H}), 7.15,7.18$, and $7.23(3 \mathrm{~m}, 1+1+2 \mathrm{H}) ;{ }^{13} \mathrm{C} \mathrm{NMR}\left(100.6 \mathrm{MHz}, \mathrm{CDCl}_{3}\right) \delta 28.10\left(21-\mathrm{CH}_{3}\right), 28.36$ $\left(\mathrm{CMe}_{3}\right), 30.87\left(\mathrm{CMe}_{3}\right), 31.99\left(23-\mathrm{CH}_{3}\right), 47.64\left(\mathrm{C}^{1}\right), 47.73\left(\mathrm{C}^{3}\right), 101.60\left(\mathrm{C}^{\alpha}\right), 105.13\left(\mathrm{C}^{\beta}\right), 122.50$ $\left(C^{4,7}\right), 125.62\left(C^{\gamma}\right), 127.02\left(C^{5}\right), 127.21\left(C^{6}\right), 148.38\left(C^{9}\right), 150.03\left(C^{8}\right), 172.36\left(C^{2}\right)$, assigned by comparison with 4g. Anal. Calcd for $\mathrm{C}_{20} \mathrm{H}_{26}$ (266.4): C, 90.16; H, 9.84. Found: C, 88.74; H, 10.18.

Another run in pure $E t_{2} O$ with $2 \mathbf{a}(0.26 \mathrm{M}), t-\mathrm{BuC} \equiv \mathrm{CLi}(0.52 \mathrm{M})$, and $\mathrm{MeLi}(0.08 \mathrm{M}$ plus $0.065 \mathrm{M}$ in two batches) furnished a similar product mixture within eight days at room temperature: $22 \%$ of $\mathbf{S 1 3}, 17 \%$ of $\mathbf{4 d}, 6 \%$ of residual $\mathbf{2 a}$, and $0.08 \mathrm{mmol}$ of the pure acid $\mathbf{S 1 2}$.

\subsection{Using Reagent 2c.}

\section{Scheme S4}

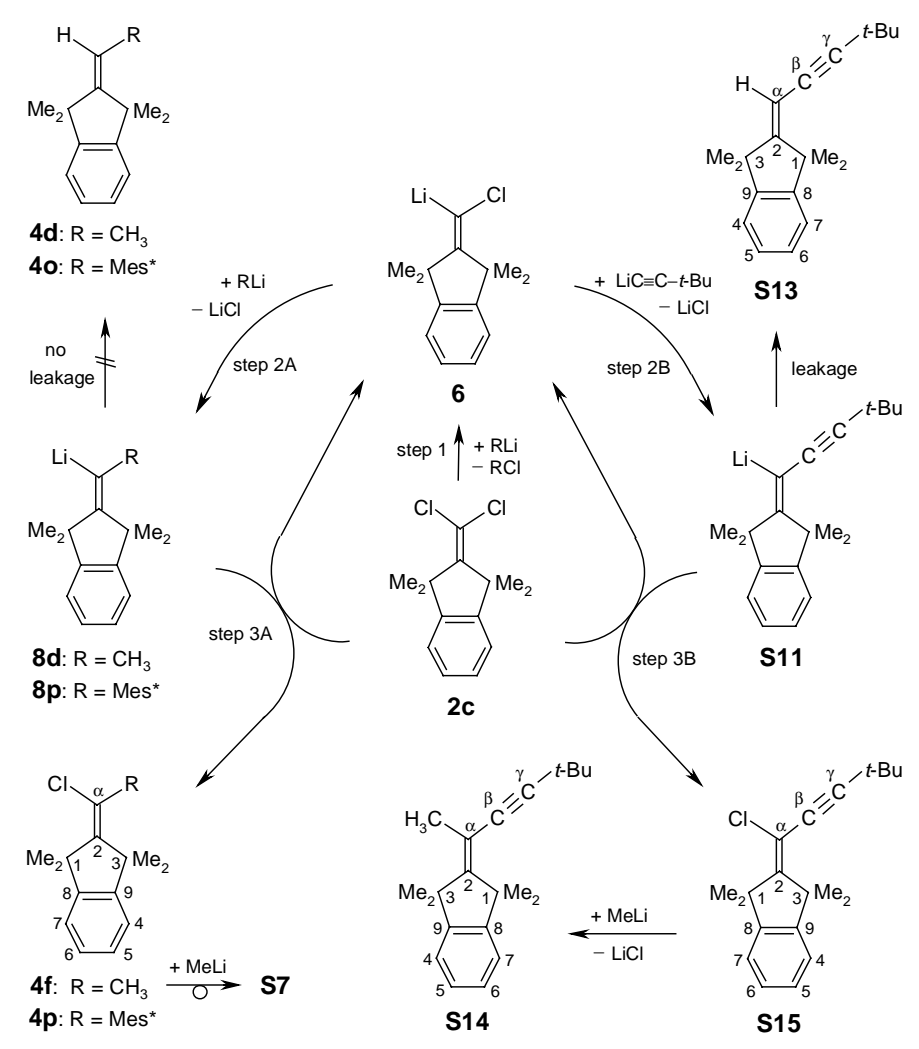


The carbenoid chain process of reagent $2 \mathrm{c}$ with $t$-BuC $\equiv \mathrm{CLi}$ (right-hand side of Scheme S4) did not occur without an initiator. It proceeded most cleanly when started (step 1) with 2,4,6-tri-tertbutylphenyllithium $\left(\mathrm{RLi}=\right.$ LiMes $\left.^{*}\right){ }^{43}$ furnishing $\mathbf{S 1 5}$ as the main product together with $\mathbf{4 p}$ but practically no leakage products $\mathbf{S 1 3}$ and 4o. The initiator RLi = MeLi behaved less nicely because it tended to modify the products $\mathbf{S 1 5}$ and $\mathbf{4 f}$ of both chains B and A: S15 (but not $\mathbf{4 f}$ ) was substituted slowly at reaction time to give $\mathbf{S 1 4}$; this necessitated to employ a larger than catalytic amount of MeLi, with the consequence of a significant contribution of the MeLi chain process (A). The much slower conversion of $\mathbf{4 f}$ led to the alkyne $\mathbf{S 7}$, as detailed in Scheme S2.

2-(1,4,4-Trimethyl-2-pentyn-1-ylidene)-1,1,3,3-tetramethylindan (S14). The sluggish conversion of $t$-BuC $\equiv \mathrm{CLi}(0.75 \mathrm{mmol}, 0.60 \mathrm{M})$ and reagent $2 \mathrm{c}(0.40 \mathrm{mmol})$ in $\mathrm{Et}_{2} \mathrm{O}$ plus THF (72:30 by volume) at room temperature had to be started and restarted with three portions $(3 \times 0.08 \mathrm{mmol})$ of $\mathrm{MeLi}$ in $\mathrm{Et}_{2} \mathrm{O}$ and a final batch of MeLi $(0.62 \mathrm{mmol})$ in the course of six days until $2 \mathrm{c}$ was consumed. The mixture was poured onto solid $\mathrm{CO}_{2}$, warmed up, and dissolved in $\mathrm{Et}_{2} \mathrm{O}$ plus $2 \mathrm{M} \mathrm{NaOH}$. The $\mathrm{Et}_{2} \mathrm{O}$ extracts of the acidified $\mathrm{NaOH}$ layer were washed until neutral, dried over $\mathrm{Na}_{2} \mathrm{SO}_{4}$, and concentrated to furnish pure $t-\mathrm{BuC} \equiv \mathrm{CCO}_{2} \mathrm{H}(\mathbf{S 1 2}, 64 \mathrm{mg}, 0.51 \mathrm{mmol})$ which crystallized after several days with $\mathrm{mp} 44-46{ }^{\circ} \mathrm{C}$ (ref S23: 47-48 $\left.{ }^{\circ} \mathrm{C}\right) ;{ }^{1} \mathrm{H}$ NMR $\left(200 \mathrm{MHz}, \mathrm{CDCl}_{3}\right) \delta 1.30(\mathrm{~s}, \mathrm{CMe}$ ) $), 8.0(\mathrm{OH})$. The ethereal layer with the non-acidic fraction was washed until neutral, dried over $\mathrm{MgSO}_{4}$, and concentrated to give $111 \mathrm{mg}$ of a yellow oil containing 4f, S14, and S15 in the molar ratio 25:47:28. Prolonged treatment with MeLi in $\mathrm{Et}_{2} \mathrm{O} / \mathrm{THF}(1: 1)$ destroyed $\mathbf{4 f}$ (section 4 ) and converted S15 to S14. After carboxylation and removal of the acids (S9, S10), this non-acidic fraction was distilled at $135-145^{\circ} \mathrm{C}$ (bath temperature)/2 mbar to afford $\mathbf{S 1 4}$ as a colorless oil: ${ }^{1} \mathrm{H}$ NMR $\left(400 \mathrm{MHz}, \mathrm{CDCl}_{3}\right) \delta 1.30\left(\mathrm{~s}, \mathrm{CMe}_{3}\right), 1.50\left(\mathrm{~s}, 23-\mathrm{CH}_{3}\right), 1.64$ (s, $\left.21-\mathrm{CH}_{3}\right), 2.07\left(\mathrm{~s}, \alpha-\mathrm{CH}_{3}\right), 7.13(\mathrm{~m}, 4-\mathrm{H}), 7.15(\mathrm{~m}, 7-\mathrm{H})$, and $7.22(\mathrm{~m}, 5-/ 6-\mathrm{H})$, assigned by the NOESY correlations $\alpha-\mathrm{CH}_{3} \leftrightarrow 3-\mathrm{CH}_{3} \leftrightarrow 4-\mathrm{H}$ and $1-\mathrm{CH}_{3} \leftrightarrow 7-\mathrm{H} ;{ }^{13} \mathrm{C}$ NMR $\left(100.6 \mathrm{MHz}, \mathrm{CDCl}_{3}\right) \delta$ $21.69\left(\operatorname{sharp~q},{ }^{1} J=127.8 \mathrm{~Hz}, \alpha-\mathrm{CH}_{3}\right), 28.18\left(\mathrm{~m},{ }^{2} J=4 \mathrm{~Hz}, C \mathrm{Me}_{3}\right), 28.43\left({ }^{1} J=126.7 \mathrm{~Hz},{ }^{3} J=4.4 \mathrm{~Hz}\right.$, $\left.21-\mathrm{CH}_{3}\right), 29.18\left({ }^{1} J=126.7 \mathrm{~Hz},{ }^{3} J=4.4 \mathrm{~Hz}, 23-\mathrm{CH}_{3}\right), 30.84\left(\mathrm{qm},{ }^{1} J=127.0 \mathrm{~Hz},{ }^{3} J=4.6 \mathrm{~Hz}, \mathrm{C} M e_{3}\right)$, $47.82\left(\mathrm{~m}, \mathrm{C}^{3}\right), 48.24\left(\mathrm{~m}, \mathrm{C}^{1}\right), 81.78\left(\mathrm{q},{ }^{3} J=5.3 \mathrm{~Hz}, \mathrm{C}^{\beta}\right), 102.48\left(\mathrm{~m},{ }^{3} J=5.1 \mathrm{~Hz}, \mathrm{C}^{\gamma}\right), 112.15\left(\mathrm{q},{ }^{2} J=\right.$ $\left.6.3 \mathrm{~Hz}, \mathrm{C}^{\alpha}\right), 122.21$ and $122.42\left(2 \mathrm{dm}\right.$, both $\left.{ }^{1} J=156 \mathrm{~Hz}, \mathrm{C}^{4,7}\right), 126.87$ and $126.96\left(2 \mathrm{dm}\right.$, both ${ }^{1} J=159$ $\left.\mathrm{Hz}, \mathrm{C}^{5,6}\right), 150.06\left(\mathrm{~m}, \mathrm{C}^{9}\right), 150.25\left(\mathrm{~m}, \mathrm{C}^{8}\right), 162.41\left(\mathrm{~m}, \mathrm{C}^{2}\right)$, assigned by selective $\left\{{ }^{1} \mathrm{H}\right\}$ decoupling as follows: $\left\{\mathrm{CMe}_{3}\right\} \rightarrow \mathrm{CMe}_{3}(\mathrm{~s})$ and $\mathrm{C}^{\gamma}(\mathrm{s}),\left\{3-\mathrm{CH}_{3}\right\} \rightarrow \mathrm{C}^{3}(\mathrm{~s})$ and $\mathrm{C}^{9}\left(\mathrm{t},{ }^{3} \mathrm{~J}=6.2 \mathrm{~Hz}\right),\left\{1-\mathrm{CH}_{3}\right\} \rightarrow \mathrm{C}^{1}(\mathrm{~s})$ and $\mathrm{C}^{8}\left(\mathrm{t},{ }^{3} J=6 \mathrm{~Hz}\right),\left\{\alpha-\mathrm{CH}_{3}\right\} \rightarrow \mathrm{C}^{\alpha}(\mathrm{s})$ and $\mathrm{C}^{\beta}(\mathrm{s}) ; \mathrm{IR}(\mathrm{KBr}) 2965,2926,2864,1590(\mathrm{w}), 1485$, 1458, 1362, 1022, and $755 \mathrm{~cm}^{-1}$. Anal. Calcd for $\mathrm{C}_{21} \mathrm{H}_{28}(280.5)$ : C, 89.94; H, 10.06. Found: C, $88.19 ; \mathrm{H}, 9.98$. 
2-(1-Chloro-4,4-dimethyl-2-pentyn-1-ylidene)-1,1,3,3-tetramethylindan (S15). The reaction of $t$ -

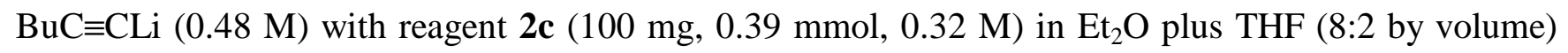
was started with LiMes* $(\mathbf{2 1 p}, 0.067 \mathrm{M})$ whose concentration dropped to $66 \%$ in the course of $115 \mathrm{~min}$ at room temperature. After two restarts with LiMes* over the next days, the mixture was poured onto solid $\mathrm{CO}_{2}$, warmed up to room temperature, and dissolved in $\mathrm{Et}_{2} \mathrm{O}$ plus $2 \mathrm{M} \mathrm{NaOH}$. The acidified $\mathrm{NaOH}$ phase afforded pure $t-\mathrm{BuC} \equiv \mathrm{CCO}_{2} \mathrm{H}^{\mathrm{S} 23}(\mathbf{S 1 2}, 11 \mathrm{mg}, 0.09 \mathrm{mmol})$. The non-acidic fraction (148 mg) was a mixture of S15 (0.20 mmol, 71\% yield), 4p (0.033 mmol, $12 \%$ yield), ClMes* (0.064 mmol), HMes* $(0.110 \mathrm{mmol})$, residual $2 \mathrm{c}(0.109 \mathrm{mmol})$, and not more than traces of the leakage products S13 and 4o. Pure S15 was obtained through chromatography on $\mathrm{SiO}_{2}$ (petroleum ether, then $\mathrm{Et}_{2} \mathrm{O}$ ) and subsequent distillation at $140-160{ }^{\circ} \mathrm{C}$ (bath temperature)/2 mbar, followed by crystallization from methanol: $\mathrm{mp} \mathrm{88-89}{ }^{\circ} \mathrm{C} ;{ }^{1} \mathrm{H}$ NMR $\left(400 \mathrm{MHz}, \mathrm{CDCl}_{3}\right) \delta 1.32\left(\mathrm{~s}, \mathrm{CM} e_{3}\right), 1.61$ and $1.63(2 \mathrm{~s}, 2+$ 2 1-/3- $\left.\mathrm{CH}_{3}\right), 7.15(\mathrm{~m}, 4-/ 7-\mathrm{H})$, and $7.24(\mathrm{~m}, 5-/ 6-\mathrm{H}) ;{ }^{13} \mathrm{C} \mathrm{NMR}\left(100.6 \mathrm{MHz}, \mathrm{CDCl}_{3}\right) \delta 27.42$ and $27.99\left(2+21-/ 3-\mathrm{CH}_{3}\right), 28.27\left(\mathrm{CMe}_{3}\right), 30.37\left(\mathrm{CMe}_{3}\right), 49.27$ and $49.90\left(\mathrm{C}^{1,3}\right), 103.96\left(\mathrm{C}^{\gamma}\right), 109.42\left(\mathrm{C}^{\beta}\right)$, $120.31\left(C^{\alpha}\right), 122.34$ and $122.35\left(C^{4,7}\right), 127.31$ and $127.33\left(C^{5,6}\right), 148.94$ and $149.17\left(C^{8,9}\right), 162.61\left(C^{2}\right)$, assigned by comparison with 4h; IR (KBr) 2960, 2924, 2863, 2214 (w, C $\equiv \mathrm{C}), 1485,1460,1360$, 1266, 850, and $750 \mathrm{~cm}^{-1}$; $\mathrm{MS}(70 \mathrm{eV}) \mathrm{m} / \mathrm{z}(\%) 302.2\left(1.5, \mathrm{M}^{+}\right), 300.2\left(5.2, \mathrm{M}^{+}\right), 287.1(13), 285.1$ (41), 265.2 (100, $\mathrm{M}^{+}-\mathrm{Cl}$ ). Anal. Calcd for $\mathrm{C}_{20} \mathrm{H}_{25} \mathrm{Cl}$ (300.9): C, 79.84; H, 8.38; Cl, 11.79. Found: C, 80.25; H, 8.50.

\section{Kinetic H/D Isotope Effect in the Competition of Reagents $2 \mathrm{a}$ and $2 \mathrm{~b}$ for MeLi.}

A solution of the deuterated (2b) and the unlabeled monochloride (2a) in anhydrous THF (0.400 mL) and $\left[\mathrm{D}_{12}\right]$-cyclohexane ("lock" signal, $0.080 \mathrm{~mL}$ ) was prepared in an NMR tube $(5 \mathrm{~mm})$ and analyzed by ${ }^{13} \mathrm{C}$ NMR at maximum resolution: The initial concentrations $[\mathbf{2 a}]_{\mathrm{o}}=0.061 \mathrm{M}$ and $[\mathbf{2 b}]_{\mathrm{o}}=0.056 \mathrm{M}$ were found through integration of isotope-shifted, baseline-separated signals of $\mathrm{C}^{1}$ and $\mathrm{C}^{3}$ of $\mathbf{2 a}$ and $\mathbf{2 b}$. MeLi (1.24 $\mathrm{M}$ in $\left.\mathrm{Et}_{2} \mathrm{O}\right)$ was added under argon cover gas in an amount $(0.040 \mathrm{~mL})$ that would have made up the concentration to $[\mathrm{MeLi}]_{\mathrm{o}} \leq 0.08 \mathrm{M}$. After the rapid consumption of MeLi, the mixture was analyzed again to provide the new concentrations $[\mathbf{2 a}]_{\infty}=0.030 \mathrm{M},[\mathbf{2} \mathbf{b}]_{\infty}=0.053 \mathrm{M},[\mathbf{4} \mathbf{d}]_{\infty}=$ $0.0162 \mathrm{M}$, and $[\mathbf{4 e}]_{\infty}=0.0018 \mathrm{M}$, the latter two by integration of their isotope-shifted $\mathrm{C}^{1}$ and $\mathrm{C}^{\beta}$ resonances. The rate ratio $k_{\mathrm{H}} / k_{\mathrm{D}}=11 \pm 4$ was obtained as the average of $k_{\mathrm{H}} / k_{\mathrm{D}}=\left\{\ln [\mathbf{2 a}]_{\mathrm{o}}-\right.$ $\left.\ln [\mathbf{2 a}]_{\infty}\right\} /\left\{\ln [\mathbf{2} \mathbf{b}]_{\mathrm{o}}-\ln [\mathbf{2} \mathbf{b}]_{\infty}\right\} \quad$ and $\quad k_{\mathrm{H}} / k_{\mathrm{D}}=\left\{\ln [\mathbf{2} \mathbf{a}]_{\mathrm{o}}-\ln \left([\mathbf{2} \mathbf{a}]_{\mathrm{o}}-\ln [\mathbf{4} \mathbf{d}]_{\infty}\right)\right\} /\left\{\ln [\mathbf{2} \mathbf{b}]_{\mathrm{o}}-\ln \left([\mathbf{2} \mathbf{b}]_{\mathrm{o}}-\right.\right.$ $\left.\left.\ln [\mathbf{4 e}]_{\infty}\right)\right\}$ without corrections for the slight dilution and the leakage protonation. After a second 
addition corresponding to $[\mathrm{MeLi}]=0.060 \mathrm{M}$, a third in situ analysis revealed that 2a had disappeared completely, leaving an excess of $\mathbf{2 b}$. Upon carboxylative workup (no organic acids found), a high

resolution ${ }^{13} \mathrm{C}$ NMR analysis showed the final distribution of $\mathbf{2 a}, \mathbf{2 b}, \mathbf{4 d}$, and $\mathbf{4 e}$ to be 0:39:43:18, confirming a high isotope effect through the total consumption of $\mathbf{2 a}$.

\section{References}

( $\neq$ ) Sterically Congested Molecules, 18; for Part 17, see: Böhrer, G.; Knorr, R.; Böhrer, P.; Schubert, B. Liebigs Ann./Recueil 1997, 193-202.

(8) Knorr, R.; Mehlstäubl, J.; Böhrer, P. Chem. Ber. 1989, 122, 1791-1793 and refs therein.

(9) Knorr, R.; Freudenreich, J.; von Roman, T.; Mehlstäubl, J.; Böhrer, P. Tetrahedron 1993, 49, $8837-8854$.

(16) All $t_{1 / 2}$ values in this work are meant to convey semiquantitative rate information only and to point at primary kinetic isotope effects in reactions of $\mathbf{2} \mathbf{b}$. They cannot be used directly to quantitate $k_{\mathrm{H}} / k_{\mathrm{D}}$ ratios because $t_{1 / 2}$ increases with decreasing initial concentrations [RLi] which varied by factors up to three here.

(43) Bauer, W.; Winchester, W.; Schleyer, P. v. R. Organometallics 1987, 6, 2371-2379.

(46) Knorr, R.; Hoang, T. P.; Nöth, H.; Linti, G. Organometallics 1992, 11, 2669-2673.

(47) Knorr, R.; Lattke, E.; Räpple, E. Liebigs Ann. Chem. 1980, 1207-1215.

(52) Watanabe, S.; Kawashima, T.; Tokitoh, N.; Okazaki, R. Bull. Chem. Soc. Jpn. 1995, 68, 1437-1448.

(58) Knorr, R.; von Roman, T.; Freudenreich, J.; Hoang, T. P.; Mehlstäubl, J.; Böhrer, P.;

Stephenson, D. S.; Huber, H.; Schubert, B. Magn. Reson. Chem. 1993, 31, 557-565.

(S1) Solvent mixture of Weidenbruch, M.; Kramer, K. J. Organomet. Chem. 1985, 291, 159-163.

(S2) Reimann, E.; Langwieler, I. Arch. Pharm. 1975, 308, 889-892.

(S3) Tour, J. M.; Stephens, E. B. J. Am. Chem. Soc. 1991, 113, 2309-2311.

(S4) Lamba, J. J. S.; Tour, J. M. J. Am. Chem. Soc. 1994, 116, 11723-11736, on p 11734.

(S5) Gilman, H.; Brook, A. G.; Miller, L. S. J. Am. Chem. Soc. 1953, 75, 3757-3759.

(S6) Burkhard, C. A. J. Am. Chem. Soc. 1946, 68, 2103-2103.

(S7) Torsell, K.; Goldman, J.; Petersen, T. E. Liebigs Ann. Chem. 1973, 231-240.

(S8) Modification of a procedure described by: Knorr, R.; Ruhdorfer, J.; Böhrer, P.; Bronberger, H.; Räpple, E. Liebigs Ann. Chem. 1994, 433-438.

(S9) Rieker, A.; Niederer, P. Chem. Ber. 1969, 102, 3947-3949, and refs therein. 
(S10) Yoshifuji, M.; Tanaka, S.; Inamoto, N. Bull. Chem. Soc. Jpn. 1975, 48, 2607-2608, and refs therein.

(S11) Märkl, G.; Merz, A. Tetrahedron Lett. 1971, 1269-1272.

(S12) Shibata, K.; Itoh, Y.; Tokitoh, N.; Okazaki, R.; Inamoto, N. Bull. Chem. Soc. Jpn. 1991, 64, 3749-3751.

(S13) Glaser, C. Ann. Chem. 1870, 154, 137-171, on p 141.

(S14) Compound 25 in ref 58.

(S15) Brunton, G.; Griller, D.; Barclay, L. R. C.; Ingold, K. U. J. Am. Chem. Soc. 1976, 98, 68036811.

(S16) Hafner, K. Pure Appl. Chem. 1990, 62, 531-540, on p 535.

(S17) Jaffe, F. J. Organomet. Chem. 1970, 23, 53-62.

(S18) Creary, X. J. Am. Chem. Soc. 1977, 99, 7632-7639.

(S19) Klein, J.; Becker, J. Y. Tetrahedron 1972, 28, 5385-5392.

(S20) Taherirastgar, F.; Brandsma, L. Chem. Ber./Recueil 1997, 130, 45-48.

(S21) Maercker, A.; Fischenich, J. Tetrahedron 1995, 51, 10209-10218.

(S22) Ludvig, M. M.; Lagow, R. J. J. Org. Chem. 1990, 55, 4880-4883.

(S23) Moureu, C.; Delange, R. Bull. Soc. Chim. Fr. 1903, 648-662, on p 654. 